\title{
Efficiency of Large Double Auctions
}

\author{
Martin W. Cripps and Jeroen M. Swinkels* \\ John M. Olin School of Business \\ Washington University in St. Louis
}

October, 2003

\begin{abstract}
We consider large double auctions with private values. Values need be neither symmetric nor independent. Multiple units may be owned or desired. Participation may be stochastic. We introduce a very mild notion of "a little independence." We prove that all non-trivial equilibria which satisfy this notion are asymptotically efficient. For any $\alpha>0$, inefficiency disappears at rate $1 / n^{2-\alpha}$.
\end{abstract}

\section{Introduction}

Many market settings are approximated by a double auction. Standard examples are the London gold market, and the order books maintained by NYSE specialists. These auctions typically have many traders on each side of the market.

More importantly, large double auctions are in some sense the "right" model for micro-foundations of price formation in competitive markets. Like a competitive market, a large double auction has many traders. However, unlike the standard competitive model, traders are strategic. Hence, if traders asymptotically ignore their effect on price this is a result, not an assumption. And, there is an explicit mechanism translating individual behaviors into prices. So, one of the thorniest problems of the standard Walrasian model - how does the market get to equilibrium if everyone is a price taker - is explicitly addressed. Finally, double auctions are a better setting for thinking about price formation than one-sided auctions, both because they are often a better match to reality, and especially because they capture the essential problems of trade better than a one-sided auction. A large one sided auction allows one to ask if traded units end up in the right hands. But, it does not address whether the correct number of units trade in the first place.

${ }^{*}$ We thank, without implicating, George Mailath, Andy McLennan, Rich McLean, John Nachbar, Phil Reny, and Mark Satterthwaite. We also thank the Boeing Center for Technology, Information, and Manufacturing for financial support. 
In a seminal paper, Rustichini, Satterthwaite and Williams (1994, henceforth RSW) consider a double auction in which $n$ buyers and sellers draw private values iid. They show that symmetric, increasing, differentiable equilibria in this setting are in the limit efficient and that convergence is fast, of order $1 / n^{2}$. This is especially attractive in light of experimental evidence on efficiency in double auctions with only a moderate number of players. ${ }^{1}$

Fudenberg, Mobius and Seidel (2003) extend the convergence result (without a rate) to a setting in which a one dimensional state is sampled and values are then drawn iid from a density that depends on the state, but has non-shifting support and uniform lower bound across states. ${ }^{2}$ They also show existence of a pure increasing symmetric equilibrium when the number of players is large.

These results are useful in thinking about how auctions approximate competitive equilibria. However, there are several dimensions along which they could be strengthened.

1. The proof technique depends heavily on symmetric distributions of values.

2. Even in the symmetric setting, there is no guarantee of uniqueness. So, while well behaved symmetric equilibria are asymptotically efficient, there may be other (possibly asymmetric) equilibria as well. In particular, there is always the no-trade equilibrium in which all buyers make an offer of zero, and all sellers make an offer higher than any possible valuation. Results before this paper do not rule out other intermediate trade equilibria.

3. While one may be willing to rule out the asymmetric equilibria on a priori grounds in the symmetric case, selecting the "good" equilibria is much harder if the initial setting is itself asymmetric.

4. Imposing symmetry on values and bids assumes away half the problem. Objects that trade automatically move from and to the right people, and so the only question is whether the volume of trade is right. Without symmetry, it may also occur that, for example, a low valued buyer wins an object when a higher valued buyer does not.

5. Finally, these papers consider only single unit demands and supplies.

We present a model and results addressing all these points. We consider a generalized private value double auction setting. ${ }^{3}$ Players can be highly asym-

\footnotetext{
${ }^{1}$ Satterthwaite and Williams (2002) establish that in the iid setting, this rate is fastest among all mechanisms. Important precursors to RSW include Chat terjee and Samuelson (1983), Wilson (1985), Gresik and Satterthwaite (1989), and Satterthwaite and Williams, (1989).

${ }^{2}$ Our model will encompass this case. See Example 4 below.

${ }^{3}$ A beautiful paper by Perry and Reny (2003) extends the previous work on information aggregation in large one sided common value auctions (Wilson 1977, Milgrom 1979, Pesendorfer and Swinkels 1997, etc.) to the double auction setting. A symmetric single unit demand and supply setting is maintained. Using a discrete bid space to get existence, they show that in a one dimensional affiliated setting, the equilibrium price converges to the rational expectations equilibrium value. So, Perry and Reny generalizes RSW in the direction of non-private values while retaining most of other restrictions, while we generalize RSW in most other directions, while, critically, retaining private values.
} 
metric, and demand or supply multiple units. Beyond the assumption of private values, there are only three assumptions with any bite. First, while individual values need be neither full support or even non-degenerate, we require that any given interval in the support of values is eventually hit in expectation by many players. We term this condition no asymptotic gaps (NAG). Analogously, we will require there to be no asymptotic atoms ( $N A A$ ): it cannot be the case that a positive limiting fraction of players are expected to pile up in the same arbitrarily small interval.

Most critically, we drastically relax independence. We require only that a "little" independence across players persists as the number of players grows. A sequence of distributions over player values satisfies $z$-independence, $z \in(0,1]$ if the probability of any given event on player $i$ 's values changes by factor bounded between $z$ and $1 / z$ when one conditions on the values of the remaining players, where $z$ holds uniformly in the number of players. 1-independence is the standard notion of independence, while two perfectly correlated random variables do not satisfy $z$-independence for any $z>0$.

An interpretation of $z$-independence is that each player has at least a small idiosyncratic component to his valuation, one that cannot be precisely predicted no matter how much one knows about the values of other players. As such, this is a fairly weak condition, admitting very broad classes of distributions.

Because values can be highly correlated (positively, negatively or otherwise) under $z$-independence, even in the limit the allocation and price setting problem will generally be non-trivial.

There is always a no-trade equilibrium in a double auction setting. Jackson and Swinkels (2001, henceforth JS) show that there is at least one non-trivial equilibrium as well. Our major result is simply stated:

As the number of players grows, every non-trivial equilibrium of the double auction setting converges to the Walrasian outcome. Inefficiency disappears at rate $1 / n^{2-\alpha}$ for any $\alpha>0$.

Asymptotic efficiency implies asymptotic uniqueness and pureness: over relevant ranges, bids must be arbitrarily close to value. Thus, as $n$ grows large, there are precisely two types of equilibria of private value double auctions:

1. equilibria involving no trade

2. equilibria in which a near efficient level of trade occurs, at a price near the competitive one.

With single unit demands and supplies, our proof works because in each outcome of a double auction, there is at most one buyer who is both currently winning an object and who would have raised the price had he bid more (the lowest winning buyer). So, while many buyers might have raised price by bidding more, only one would care that he did so. This is symmetric for sellers considering lowering their bids. So, the expected relevant impact on price from increased bids by buyers is already order $1 / n$. And, with lots of bidders, even 
if an increase in bid increases price, it should do so by an amount related to $1 / n$, since this should be the expected distance to the next bid. But then, since the expected impact on price is order $1 / n^{2}$, it must be that bidding honestly almost never wins an extra object, and so those objects that are traded must be allocated very efficiently.

The focus then turns to showing that the right number of objects trade, or, equivalently, that the competitive gap defining the range of market clearing prices grows small. This turns out to be much the hardest part of the paper (especially with a rate). In the symmetric case, one can appeal to the first order conditions of players near a discontinuity in bids. Here, things are much more difficult, as without symmetric increasing strategies, (a) the very concept of a "gap" becomes more complicated (b) it is hard to identify which player types might bid near a gap, and (c) players can have very different beliefs about the likelihoods of the events involved. We show that the only way to have a significant competitive gap without violating the efficiency already shown for those objects traded is for the market to essentially become deterministic, with a given set of buyers and sellers always trading. But then, any member of either of these groups can favorably influence the price without losing the chance to trade.

The efficiency result generalizes to multiple unit demands as long as $N A G$ continues to hold for the first unit of demand and supply for each player. If this holds, we can reformulate the arguments just outlined applied only to the highest bid by each buyer and lowest bid by each seller to show small price impacts of honest bidding. From there to (fast) efficiency for all units involves a careful tracking of incentives, but is otherwise straightforward.

We begin by setting up the basic single unit demand and supply model. We then introduce $z$-independence. Analysis of efficiency for the large double auction with single unit demands and supplies follows. Then, we generalize to auctions with multiple unit demands and supplies. We conclude with some thoughts on extensions. All proofs are relegated to an appendix.

\section{The Model}

We begin with the structure of a given double auction $\mathcal{A}$. A set finite set $N$ of players is divided into subsets $N_{S}$ and $N_{B}$. Players in $N_{S}$ are potential sellers, each with one unit to sell. Players in $N_{B}$ are potential buyers, each desiring a single unit.

Each $i \in N$ has valuation $v_{i}$. For sellers, this might be either a production cost or a value in use. For $i \in N_{B}$, we assume $v_{i} \in[0,1)$. For $i \in N_{S}$, we assume $v_{i} \in(0,1]$. A buyer with value 0 or a seller with value of 1 will never trade. Because of this, there is no loss of generality in assuming an equal number of buyers and sellers. Let $n \equiv\left|N_{s}\right|=\left|N_{B}\right|$. Because one can "park" extra buyers at 0 and extra sellers at 1 , the model also allows a stochastic number of buyers and sellers. The vector $v \equiv\left\{v_{i}\right\}_{i \in N}$ is drawn according to a probability measure $P$ on $[0,1)^{n} \times(0,1]^{n}$. The marginal of $P$ onto $v_{i}$ is $P_{i}$. 
Each player $i$ observes his value and then submits a bid $b_{i} \in[0,1]$. Trade is determined by crossing the demand and supply curves constructed from the submitted buy and sell bids. ${ }^{4}$ Call the (random) range of possible market clearing prices the competitive gap, $c g \equiv[c g, \overline{c g}]$. If we let $b^{(i)}$ denote the $i^{\text {th }}$ highest bid, then a little time with the appropriate figure shows that $c g=\left[b^{n+1}, b^{n}\right]$.

Assumption 1 Trade takes place at price

$$
p=\hat{p}(\underline{c g}, \overline{c g})
$$

where $\hat{p}$ is differentiable, takes values in $[\underline{c g}, \overline{c g}]$, and has derivatives bounded by 0 and $1 .^{5}$

Imagine that the bidder who submitted $\overline{c g}$ raises his bid substantially. As long as his bid continues to define $\overline{c g}$, he raises the price at rate at most 1 . As soon as he passes the next bid up, he ceases to affect price. Let $\overline{u g}=b^{n-1}$ be this next bid, and define the upper supporting gap as $u g \equiv[\overline{c g}, \overline{s g}]$. Then, the maximum effect on the price is $|u g|$. Similarly, let $\underline{l g} \equiv b^{n+1}$, and define the lower supporting gap as $l g \equiv[l g, c g]$. So, $c g$ determines the amount of choice there is in setting a market price, while $l g$ and $u g$ determine how closely "supported" this range is.

Each player $i$ has a $v N M$ utility function $u_{i}$. No particular structure on risk preferences is required, but we do require each $u_{i}$ to be increasing and have slope bounded from 0 and $\infty .^{6}$

\subsection{Equilibrium}

A set of distributional strategies $\left\{\mu_{i}\right\}_{i \in N}$ (Milgrom and Weber, 1982) is an equilibrium if it is a Bayesian Nash equilibrium in which buyers never bid above $v_{i}$, and sellers never bid below $v_{i}$. The equilibrium is non-trivial if there is a positive probability of trade.

We show that non-trivial equilibria are asymptotically efficient. This, of course, is a better result if such equilibria exist! Under slightly stronger conditions than we use here, JS show that this is indeed the case. ${ }^{7}$

\subsection{Sequences of Auctions}

Consider a sequence of such auctions $\left\{\mathcal{A}^{n}\right\}$, where $n$ tends to infinity. We need three conditions that apply across $n$. First, while individual values need not have full support (and may, in fact, be atomic), we require that as $n$ grows large, each subinterval is hit with non-vanishing probability.

\footnotetext{
${ }^{4}$ If tied buy and sell bids allow more than one level of trade, the largest is chosen.

${ }^{5}$ This of course includes the standard $k$ double auction.

${ }^{6}$ In the proofs, we assume risk neutrality. Dealing with $v N M$ utility functions with slope bounded from 0 and $\infty$ involves scaling potential gains down by some factor from the risk neutral case, and potential loses up. This merely introduces notation.

${ }^{7}$ The two key assumptions are mutual absolute continuity of $P$ with respect to $\Pi_{i} P_{i}$, and atomless $P_{i}$. Neither assumption plays any further role in the development here.
} 
Assumption 2 (No Asymptotic Gaps) There is $w>0$ such that for all $n$, and for all intervals $I \subseteq(0,1)$ of length $1 / n$ or greater,

$$
\sum_{i \in N_{B}} P_{i}[I] \geq w n|I|
$$

and

$$
\sum_{i \in N_{S}} P_{i}[I] \geq w n|I| .
$$

Note that $P, N_{B}, N_{S}$ etc. all vary from one $\mathcal{A}^{n}$ to another. We suppress this in our notation as convenient.

Our second assumption is similar:

Assumption 3 (No Asymptotic Atoms) There is $W<\infty$ such that for all $n$, and for all intervals $I \subseteq(0,1)$ of length $1 / n$ or greater,

$$
\sum_{i \in N_{B}} P_{i}[I] \leq W n|I|
$$

and

$$
\sum_{i \in N_{S}} P_{i}[I] \leq W n|I| .
$$

That is, not too many values fall in any given interval. These conditions hold only on $(0,1)$, allowing a positive mass of buyers with value 0 or sellers with value 1 , consistent with our earlier discussion of "parking" extra players.

Example 1 Let sellers $i \in\{1, \ldots, n\}$ have $v_{i}$ degenerate at $i / n$ and similarly for buyers. NAG and NAA are satisfied for $w=W=1$. So, individual values need neither have full support nor be non-atomic.

Example 2 Each $P_{i}$ is continuous with density bounded by $w$ and $W$.

Each of these two assumption has an analog in RSW. NAA is needed for a rate of convergence result, but not for convergence itself.

\section{$3 \quad z$-Independence}

Our final condition is the most important. We wish to relax independence considerably while still requiring "some persistent independence" as the population grows.

We require that knowledge about the values of players other than $i$ provides at most a finite likelihood ratio on the values of player $i$, independent of how many other players there are. 
Definition 1 The sequence of probability measures $\left\{P^{n}\right\}$ satisfies z-independence, $z \in(0,1]$, if for all $n$, for all $i \in N$, for any positive probability event $F_{-i}$ involving only $v_{-i}$ and any positive probability event $F_{i}$ involving only $v_{i}$,

$$
z \operatorname{Pr}\left(F_{i}\right) \leq \operatorname{Pr}\left(F_{i} \mid F_{-i}\right) \leq \frac{1}{z} \operatorname{Pr}\left(F_{i}\right) .
$$

That is, there is still some idiosyncrasy in each $v_{i}$ even as the market becomes large. ${ }^{8}$

For fixed $n, z$-independence is slightly stronger than mutual absolute continuity (consider a uniform and a triangular distribution on $[0,1]$ ) but weaker than having a continuous Radon-Nikodym derivative bounded from 0 and $\infty$. The real content of $z$-independence is in the uniformity of $z$ across $n$.

Assumption 4 (z-independence) There exists $z>0$ such that $\left\{P^{n}\right\}$ is $z$ independent.

\subsection{Examples}

Example 3 With probability $1 / 2$, players are drawn iid uniform $[0,1]$, and with probability $1 / 2, x$ is drawn uniformly from $[1 / n, 1-1 / n]$, and values are drawn iid uniform $[x-1 / n, x+1 / n]$. For each $n, P^{n}$ is absolutely continuous with respect to $\prod P_{i}$ (and, the example is easily modified such that the Radon-Nikodym derivative is continuous as well). But, as $n \rightarrow \infty$, seeing the values of two randomly selected players within $2 / n$ of each other makes it arbitrarily likely that all remaining players will also have such a value.

We would like this example to be ruled out by our notion of "some persistent independence." A first thought might be to require that no matter what we know about one subset of the players' values, beliefs about the rest of the players' values are updated by at most a finite ratio. This turns out to be much too strong.

Example 4 Nature chooses $x \in\{L, H\}$ equiprobably. If $L$ is drawn, values are drawn iid according to density $f(v)=1 / 2+v$. If $H$ is drawn, values are drawn iid according to density $f(v)=3 / 2-v$.

A finite likelihood ratio condition fails if both events involve large numbers of players. For example, let $F_{O}$ be the event that less than $50 \%$ of the odd numbered buyers have value below $1 / 2$, and let $F_{E}$ be the event that less than $50 \%$ of the even numbered buyers have value below $1 / 2$. Then, as $n \rightarrow \infty, \operatorname{Pr}\left(F_{O} \mid F_{E}\right) \rightarrow 1$, while $\operatorname{Pr}\left(F_{O} \mid F_{E}^{C}\right) \rightarrow 0$. Since for each $n, F_{E}$ and $F_{E}^{C}$ have the same size, this also means that the Radon-Nikodym derivative satisfies no uniform bound across $n$.

${ }^{8}$ A contemporaneous paper by Peters and Severinov (2002) uses a similar condition (in a different model) in a finite type setting. 
This example exhibits a great deal of independence despite the fact that likelihood ratios and Radon-Nikodym derivatives diverge. We would like to admit it.

Note that Example 3 fails $z$-independence for any $z>0$, as $v_{i}$ is, $1 / 2$ of the time, arbitrarily closely predicted by $v_{-i}$. However Example 4 satisfies .5-independence; all one can extract from $v_{-i}$ is information about whether $x$ is $L$ or $H$, which changes the density on $v_{i}$ from 1 to something between $1 / 2$ and $3 / 2$.

Example 4 generalizes to any process in which a state is sampled and then, conditional on the state, values $v_{i}$ are drawn independently from measures with non-moving support $V_{i}$ according to densities uniformly bounded (across states and $n$ ) away from zero and infinity. So our setting encompasses Fudenberg et al. (2003) (and more importantly, non-symmetric analogues to their model). However, even with symmetry, $z$-independence admits many distributions which cannot be generated in this way.

Example 5 There are 2 players. The density on values is 2 on $\left[0, \frac{1}{2}\right] \times\left[0, \frac{1}{2}\right]$, and $2 / 3$ elsewhere. This satisfies $\frac{2}{3}$-independence. One can generate this using states and conditionally-independent values. In state $\theta_{1}$ (which occurs 1 time in 3) values are uniform on $\left[0, \frac{1}{2}\right] \times\left[0, \frac{1}{2}\right]$, while in state $\theta_{2}$ they are uniform on $[0,1] \times[0,1]$. But, one cannot do so without shifting supports.

Postlewaite and Schmeidler (1986) define non-exclusivity as a situation where the information of $n-1$ players is enough to predict the relevant state of the economy. A variety of follow-on papers relax this to hold only asymptotically. ${ }^{9}$ On first view, $z$-independence is antithetical to non-exclusivity, since no matter how much is known about the rest of the players, the value of player $i$ remains uncertain. However, note that non-exclusivity refers to information about the underlying state, not to the signals players realize conditional on those states. In Example $4, v_{-i}$ is asymptotically fully informative about $L$ vs. $H$, while of bounded informativeness about $v_{i}$. Hence Example 4 can satisfy both conditions.

Example 6 Nature draws $v_{1}$ uniformly from [0,1] (this person is a "fashion leader"), and then draws subsequent players iid according to a density with support $[0,1]$ but concentrated around $v_{1}$.

Since the impact of an early draw on later draws does not vanish, $z$-independence does not imply weak mixing. It is also easy to construct sequences satisfying weak mixing under which successive draws are arbitrarily correlated, violating $z$-independence.

Example 7 A parameter $x$ is chosen from $[0,1]$. Values are drawn conditionally independently according to $f(. \mid x)$, where $f(. \mid x)$ satisfies $M L R P$ in $x$. As long as $f(. \mid 0) / f(. \mid 1)$ is uniformly bounded, z-independence is satisfied for

\footnotetext{
${ }^{9}$ A good entry point is McLean and Postlewaite (2002).
} 
$z=\min _{x} f(x \mid 0) / f(x \mid 1)$. Choose a subset of the players, and replace $v_{i}$ by $1-v_{i}$. This measure continues to satisfy z-independence, but is obviously not affiliated. So, affiliation has essentially nothing to do with the issues at hand.

We close this subsection with an example illustrating the surprising degree of correlation $z$-independence can imply. Define $[x]$ as the largest integer smaller than $x$.

Example 8 For $m \leq n$, let $\zeta_{B}(m)=\left(\begin{array}{c}n \\ m\end{array}\right)(.5)^{n}$ be the probability of $m$ heads from $n$ flips of a fair coin. ${ }^{10}$

Now, for some $0<a<1 / 2$, generate $\zeta_{C}$ from $\zeta_{B}$ by first defining $\zeta_{C}^{\prime}(m)=$ $\zeta_{B}(m) a^{|m-[n / 2]|}$, and then defining $\zeta_{C}$ from $\zeta_{C}^{\prime}$ by normalizing. Informally one makes each outcome successively further away from [n/2] more unlikely by a factor of a. Choose $m$ according to $\zeta_{C}$, choose each subset of coins of size $m$ with equal probability, and make the coins in the subset heads, and the remainder tails. When a is small, drawing exactly $[n / 2]$ heads by this process becomes very probable. ${ }^{11}$ For $a=.1$, e.g., there is an $80 \%$ chance or exactly $[n / 2]$ heads regardless of $n .^{12}$

This process satisfies $\mathrm{z}$-independence! If there are $m^{\prime}$ heads among all but coin $i$, the probability that $i$ is heads is $\operatorname{Pr}\left(m=m^{\prime}+1\right) / \operatorname{Pr}\left(m=m^{\prime}+1\right)$. By construction, this is either a or $1 / a$.

Thus, $z$-independence does not imply limit "noise." So the techniques in Mailath and Postlewaite (1990), Al-Najjar and Smorodinsky (1997), and Swinkels (2001) do not apply.

\subsection{A Preliminary Lemma}

Our first lemma shows that if values are $z$-independent then so too are bids. The intuition for this is that $b_{i}$ is a garbling of $v_{i} \cdot{ }^{13}$ It also describes the implications of $z$-independence for groups of players.

10 The example can easily be extended from coins to values in the standard domain.
${ }^{11}$ Note that

$$
\begin{aligned}
\sum_{m=0}^{r} \zeta_{C}^{\prime} & =\sum_{m=0}^{r} \zeta_{B}(m) a^{|m-r / 2|} \leq \zeta_{B}(r / 2) \sum_{m=0}^{r} a^{|m-r / 2|} \\
& \leq \zeta_{B}(r / 2)\left(1+2 \sum_{i=1}^{\infty} a^{i}\right)=\zeta_{B}(r / 2)\left(1+\frac{2 a}{1-a}\right)
\end{aligned}
$$

Thus,

$$
\zeta_{C}(n / 2) \geq \frac{\zeta_{B}(n / 2)}{\zeta_{B}(n / 2)\left(1+\frac{2 a}{1-a}\right)}=\frac{1}{\left(1+\frac{2 a}{1-a}\right)} .
$$

As $a \rightarrow 0$, this tends to 1 .

${ }^{12}$ For $a=.1$, the previous expression is equal to $\frac{1}{\left(1+\frac{2(.1)}{1-.1}\right)}=.81$.

${ }^{13}$ A related lemma appears in JS. 
Throughout the paper, for any non-empty $K \subset N$, when we write $F_{K}$ (respectively $F_{i}, F_{-i}, F_{N \backslash K}$ ), we mean an arbitrary positive probability event involving only the values or bids of the players in $K(\{i\}, N \backslash i, N \backslash K)$.

Lemma 1 Fix a non-empty $K \subset N$. Let $a=\min \{|K|,|N \backslash K|\}$. Then for all $F_{K}$, and $F_{N \backslash K}$,

$$
z^{-a} \operatorname{Pr}\left(F_{K}\right) \geq \operatorname{Pr}\left(F_{K} \mid F_{N \backslash K}\right) \geq z^{a} \operatorname{Pr}\left(F_{K}\right) .
$$

Let $X_{K}$ be a random variable that depends only on the values/bids of the players in $K$. Then:

$$
z^{-a} E\left(X_{K}\right) \geq E\left(X_{K} \mid F_{N \backslash K}\right) \geq z^{a} E\left(X_{K}\right) .
$$

When $a$ is large, these bounds are weak; for arbitrary events involving many players, likelihood ratios can explode.

\subsection{Large Deviations}

Given $K \subset N$ and events $\left\{F_{i}\right\}_{i \in K}$ let $Q_{K}$ be the number of $F_{i}$ that are true. Notice that $E\left(Q_{K}\right)=\sum_{i \in K} \operatorname{Pr}\left(F_{i}\right)$. Let us stochastically bound $Q_{K}$. Note first that for each $i$,

$$
\operatorname{Pr}\left(F_{i} \mid F_{-i}\right) \geq z \operatorname{Pr}\left(F_{i}\right) .
$$

Note also that

$$
\operatorname{Pr}\left(F_{i}^{c} \mid F_{-i}\right) \leq \frac{1}{z} \operatorname{Pr}\left(F_{i}^{c}\right)=\frac{1}{z}\left(1-\operatorname{Pr}\left(F_{i}\right)\right)
$$

and so

$$
\operatorname{Pr}\left(F_{i} \mid F_{-i}\right) \geq 1-\frac{1}{z}\left(1-\operatorname{Pr}\left(F_{i}\right)\right) .
$$

Thus,

$$
\operatorname{Pr}\left(F_{i} \mid F_{-i}\right) \geq \underline{p}_{i} \equiv \max \left\{z \operatorname{Pr}\left(F_{i}\right), 1-\frac{1}{z}\left(1-\operatorname{Pr}\left(F_{i}\right)\right)\right\} .
$$

Since this is true for all $F_{-i}$ we show that $Q_{K}$ first order stochastically dominates $|K|$ independent coins with parameters $p_{i}$.

Similarly, $|K|$ independent coins with parameters

$$
\bar{p}_{i} \equiv \min \left\{\frac{1}{z} \operatorname{Pr}\left(F_{i}\right), 1-z\left(1-\operatorname{Pr}\left(F_{i}\right)\right)\right\}
$$

stochastically dominate $Q_{K}$.

Sets of independent coins are well understood. We can apply the theory of large deviations to obtain:

Lemma 2 For all $K \subset N$ and $F_{N \backslash K}$,

$$
\begin{aligned}
\operatorname{Pr}\left(Q_{K}<\frac{z}{3} E\left(Q_{K}\right) \mid F_{N \backslash K}\right) & \leq e^{-.3 z E\left(Q_{K}\right)} \\
\operatorname{Pr}\left(Q_{K}>\frac{3}{z} E\left(Q_{K}\right) \mid F_{N \backslash K}\right) & \leq e^{-E\left(Q_{K}\right)}
\end{aligned}
$$


This casts light on Example 8. Under $z$-independence, probabilities that start in the interior of $(0,1)$ cannot be moved too far toward or away from the boundaries. But, probabilities can be moved around essentially arbitrarily within $\left(p_{i}, \bar{p}_{i}\right)$.

A useful implication of Lemma 2 is that the probability of at least one success is not drastically affected by $F_{N \backslash K \text {. }}$

\section{Corollary 1}

$$
\operatorname{Pr}\left(Q_{K} \geq 1 \mid F_{N \backslash K}\right) \geq\left(1-e^{-z}\right) \operatorname{Pr}\left(Q_{K} \geq 1\right)
$$

\subsection{Normal Realizations}

We prove convergence at rate $1 / n^{2-\alpha}$ for any given $\alpha>0$. It is convenient to fix $\alpha$ now. We will need various fudge factors along the way. Choose $\alpha_{1}, \alpha_{2}, \alpha_{3}, \alpha_{4}$ so that

$$
\alpha>\alpha_{1}>\alpha_{2}>\alpha_{3}>\alpha_{4}>2 \alpha / 3
$$

Let $w^{\prime} \equiv \frac{z}{6} w$ and $W^{\prime} \equiv \frac{6}{z} W$

Definition $2 A$ realization is normal if every interval $I \subseteq(0,1)$ of length $1 / n^{1-\alpha / 3}$ or greater has between $w^{\prime} n|I|$ and $W^{\prime} n|I|$ buyers (respectively sellers) with value in that interval.

Let $\mathcal{N}$ be the event that the realization is normal.

Say that a statement is true for $n$ sufficiently large $(n \mathrm{SL})$ to mean that there exists an $n^{*}$ depending only on the parameters such that the statement is true for all $n>n^{*}$. Then, a key implication of Lemma 2 is

Lemma 3 For all $n S L, \operatorname{Pr}(\mathcal{N}) \geq 1-1 / n^{4}$.

Together with NAG and NAA, Lemma 3 implies that the limiting realized true demand and supply curves are unlikely to have either vertical or flat sections (except at 0 for buyers and 1 for sellers). ${ }^{14}$

\section{Analysis of the Double Auction}

\subsection{Summing Deviations}

Fix an equilibrium $\mu$ of $\mathcal{A}^{n}$. Consider buyer $i$ 's distributional strategy $\mu_{i}$. A deviation for $i$ is a measurable mapping $d_{i}$ from $[0,1]^{2}$ to $[0,1]$. First $i$ draws

\footnotetext{
${ }^{14}$ We show that percentage efficiency losses are asymptotically less than $1 / n^{2-\alpha}$. As for all rate of convergence results, this does not say anything about small $n$. The construction underlying normality in particular only holds for $n$ pretty large. We use normality to sidestep a set of statistical issues related to the generality of our set-up, especially non-symmetry. There seems to be nothing in the underlying incentives being exploited that precludes much faster convergence, and our expectation would be that actual convergence is indeed very fast. RSW supplement their rate result (where the constant is again large) with numerically solved examples. Such solutions are beyond our ability in this setting.
} 
$v_{i}$ and $b_{i}$ according to $\mu_{i}$, but then she modifies her chosen bid according to $d_{i}$. Consider $d_{i}$ for which $b_{i} \leq d_{i}\left(b_{i}, v_{i}\right) \leq v_{i} \forall b_{i}, v_{i}$. That is, $i$ sometimes raises her bid, but not beyond her true value (since $\mu_{i}$ did not involve $i$ bidding more than her true value, this is coherent).

In any given realization, $d_{i}$ may have benefit $\hat{B}_{i}$ in that $i$ wins when he other wise would not have, or may have cost $\hat{C}_{i}$ that $i$ pays more when he would have already won. To formalize this, let $p$ be price under $\mu$, and $p_{d}$ the price when $i$ uses $d_{i}$. Let $W_{i}$ be the event that $i$ wins with $d_{i}$, but not without. Then

$$
B_{i} \equiv E\left(\hat{B}_{i}\right)=\operatorname{Pr}\left(W_{i}\right) E\left(v_{i}-p_{d} \mid W_{i}\right) .
$$

Let $O_{i} \subset W_{i}^{c}$ be the event that $i$ wins without $d_{i}$. Then

$$
C_{i} \equiv E\left(\hat{C}_{i}\right)=\operatorname{Pr}\left(O_{i}\right) E\left(p_{d}-p \mid O_{i}\right) .
$$

Since $\mu_{i}$ is a best response, $B_{i} \leq C_{i}$. So, given such a $d_{i}$ for each buyer,

$$
\sum_{N_{B}} B_{i} \leq \sum_{N_{B}} C_{i}
$$

Each $d_{i}$ is unilateral. But, there is nothing wrong with summing the incentive constraints implied.

Consider $\sum_{N_{B}} C_{i}$. Ex-post, $\hat{C}_{i}>0$ only if (a) trade was occurring and (b) the original $b_{i}$ was equal to $\overline{c g}$, and uniquely so. When $b_{i}>\overline{c g}$ (or is tied at $\overline{c g}$ ), increasing $b_{i}$ does not affect $p$. If $b_{i}<\overline{c g}$, increasing $b_{i}$ may increase $p$, but as $i$ was not originally winning, she is unhurt. So, there is at most one $i$ with $\hat{C}_{i}>0 .{ }^{15}$ And, as discussed above, for this $i, \hat{C}_{i} \leq|u g|$. Thus,

$$
\sum_{N_{B}} C_{i} \leq \operatorname{Pr}(T) E(|u g| \mid T) .
$$

For sellers, the same analysis applies if bids are lowered, but not below value. We have thus established:

Lemma 4 For any set $\left\{d_{i}\right\}_{i \in N_{B}}$, for which $d_{i}\left(b_{i}, v_{i}\right) \in\left[b_{i}, v_{i}\right]$ for all $\left(b_{i}, v_{i}\right)$

$$
\sum_{N_{B}} B_{i} \leq \operatorname{Pr}(T) E(|u g| \mid T) .
$$

For any set $\left\{d_{i}\right\}_{i \in N_{S}}$, for which $d_{i}\left(b_{i}, v_{i}\right) \in\left[v_{i}, b_{i}\right]$ for all $\left(b_{i}, v_{i}\right)$

$$
\sum_{N_{S}} B_{i} \leq \operatorname{Pr}(T) E(|l g| \mid T) .
$$

While easy to prove, this bound is powerful. Independent of the number of bidders, the total benefit to players of bidding more aggressively in terms of making new trades must be small in equilibrium.

\footnotetext{
${ }^{15}$ If $\overline{c g}$ is a seller's bid, no buyer is hurt by $d_{i}$.
} 


\subsection{The Probability of Trade is Bounded from Zero}

An important first step is to show that non-trivial equilibria are not "almost trivial" in the sense that trade becomes increasingly rare as $n$ grows. For each $n$, choose a non-trivial equilibrium of $\mathcal{A}^{n}$. Let $V$ be the number of objects traded and $T$ be the event that $V \neq 0$.

Our first lemma is technical.

Lemma 5 Along any subsequence, if $\frac{E(V \mid T)}{n} \nrightarrow 0$, then $\operatorname{Pr}(T) \rightarrow 1$.

Intuitively, if many players trade given $T$, then many players must occasionally be bidding in a fairly aggressive way. But then, by $z$-independence, at least a fraction of them will be doing so almost all the time. The proof is more complicated because $T$ is linked to all player's actions, and so $z$-independence does not immediately apply.

Using Lemma 5, we can show:

Proposition 1 There is $\gamma>0$ such that for all $n S L$, and all non-trivial equilibria,

$$
\operatorname{Pr}(T) \geq \gamma
$$

For intuition, think about a situation where in aggregate buyers only make a "serious" offer with some probability $\delta$ close to 0 , and symmetrically for sellers (clearly, if there is a non-vanishing probability of a serious offer on either side, trade will not disappear). Trade occurs at most $2 \delta$ of the time, since trade requires a serious offer from at least one side. Hence, by Lemma 4, the total costs to buyers (or sellers) of making more generous offers is like (has the same order as) $\delta$. But, from $z$-independence, the probability of a serious offer from one side but not the other is like $(1-\delta) \delta \cong \delta$. But, when there is a serious offer on one side but not the other, a number of bidders on the other side that grows like $n$ would have benefited by deviating to grab the serious offer. The gains are thus like $n \delta$, while costs are like $\delta$. This is a contradiction.

\subsection{Small Supporting Gaps}

We show next that the upper and lower supporting gaps shrink quickly. This proceeds in two steps. First, we show that $E(|u g|$ ) (respectively $E(|l g|)$ ) is like $1 / n$. The idea is most easily seen if for each $n, u g$ has constant length $v$. By Lemma 3, a number of buyers proportional to $n v$ will have $v_{i}$ in the top half of $u g$. At most one of these buyers is winning an object (they are not bidding above $\overline{u g}$, as bids are below value, and only one bid below $\overline{u g}$ is filled). By raising $b_{i}$ to $v-v / 4$ all but this player (acting unilaterally) would win an extra object and earn at least $v / 4$. So, $\sum B_{i} \geq n v^{2}$ (up to some constants). But by Lemma $4, \sum C_{i} \leq v$, since the one person who is hurt raises the price by at most $v$. Thus,

$$
n v^{2} \leq \sum_{N_{B}} B_{i} \leq \sum_{N_{B}} C_{i} \leq v
$$


from which $v \leq \frac{1}{n}$. The actual proof has to count for the fact that $|u g|$ is stochastic, as are the number of bidders in any given interval. Formally:

Lemma 6 For $n S L$ and all $x$,

$$
E(|u g|) \leq \frac{1}{n^{1-\alpha_{4}}}, \quad E(|\lg |) \leq \frac{1}{n^{1-\alpha_{4}}}
$$

Fix $x$, and consider $\operatorname{Pr}(|u g| \geq x)$. Consider again buyers raising $b_{i}$ to $v-x / 4$. When $|u g| \geq x$, then as above, a number of buyers like $n x$ makes gains $x / 4$, and so $\sum B_{i}=n x^{2}$ (again ignoring constants). And, $\sum C_{i} \leq E(|u g|) \leq 1 / n$ from the first step. So

$$
\operatorname{Pr}(|u g| \geq x) n x^{2} \leq \frac{1}{n}
$$

from which $\operatorname{Pr}(|u g| \geq x) \leq \frac{1}{n^{2} x^{2}}$. Formally

Lemma 7 For $n S L$ and all $x$,

$$
\operatorname{Pr}(|u g| \geq x) \leq \frac{1}{n^{2-\alpha_{3}} x^{2}}, \quad \operatorname{Pr}(|l g| \geq x) \leq \frac{1}{n^{2-\alpha_{3}} x^{2}} .
$$

For $x \geq 0$, let $L_{B}(x)$ be those buyers with values above $\overline{c g}+x$ that do not receive an object, and let $l_{B}(x) \equiv \# L_{B}(x)$. Similarly let $L_{S}(x)$ be those sellers with values below $\underline{c g}-x$ who do not sell, and let $l_{S}(x) \equiv \# L_{S}(x)$. Let

$$
S L_{B}(x) \equiv \sum_{i \in L_{B}(x)} v_{i}-\overline{c g}, \quad S L_{S}(x) \equiv \sum_{i \in L_{S}(x)} \underline{c g}-v_{i}
$$

For buyers, this is the loss in consumer surplus compared with being able to price take at $\overline{c g}$, and analogously for sellers. Lemma 6 implies that both the number of such players and the associated loss is small. The intuition again comes from considering players bidding closer to their values.

Lemma 8 For $n S L$ and for all $x$,

$$
E\left(l_{B}(x)\right) \leq \frac{1}{x n^{1-\alpha 4}}, \quad E\left(l_{S}(x)\right) \leq \frac{1}{x n^{1-\alpha 4}} .
$$

Further

$$
E\left(S L_{B}(1 / n)\right) \leq \frac{1}{n^{1-\alpha_{3}}}, \quad E\left(S L_{B}(1 / n)\right) \leq \frac{1}{n^{1-\alpha_{3}}}
$$

\subsection{Small Competitive Gaps}

Let us now turn to the competitive gap. Our key lemma:

Lemma 9 For $n S L$ and for all $x$,

$$
\operatorname{Pr}(|c g| \geq x) \leq \frac{1}{n^{2-\alpha_{2}} x^{2}} .
$$


To see the intuition for Lemma 9, consider first a fixed interval $I=(I, \bar{I})$ such that $n$ prespecified bidders always bid above $\bar{I}$ (up) and the rest always bid below $I$ (down). Then, the competitive gap will always include $I$. And, since the probability of trade is bounded away from 0 , the set of up bidders must contain a buyer, and the set of down bidders a seller. But then, by bidding $I+\varepsilon$, any up buyer can still trade and force the price near the bottom of $\underline{I}$, while by bidding $\bar{I}-\varepsilon$, any down seller can still trade and force the price near $\bar{I}$, contradicting equilibrium.

If this situation arises only in the limit then the buyer or seller occasionally loses a trade by bidding more aggressively, but this becomes unlikely. Finally (because this is what we will really need), imagine that $I$ shrinks as $n$ grows. Then, as the gain from affecting the market price shrinks, we must be careful that the loss from lost trades shrinks as well. To do this, pick a buyer whose value is not too much above $\bar{I}$, so that his value of trade was quite small, and a seller whose value was not too much below $I$. The efficiency of the allocation among buyers and sellers (Lemma 8) lets us to do this.

We show that if Lemma 9 fails, then the limit is as described. For intuition, assume there is some interval $I$ of length $x$ such that nobody ever bids in $I$, and such that $\operatorname{Pr}(I \subseteq c g)$ does not fall quickly. Let $p_{i}$ be the probability that $i$ bids up, and $q_{i}$ the probability of down. Order the players so that $p_{i}$ is increasing. Run along them stopping at the player $i$ where one counts $n-1$ ups. For $I \subseteq c g$, we need to hit exactly one more up in the rest of the sequence. If one hits no more ups, $I \subseteq u g$, while if one hits 2 more ups, $I \subseteq l g$, either of which is rare by Lemma 7. But, we argue, the only way to make 1 more up likely, but neither 0 nor 2 more ups likely is for the next player to have $p_{i+1}$ nearly 1 , and for the remaining players to in aggregate have almost no chance of even one up. Essentially, if $p_{i+1}$ is not near one, then, since $p_{i}$ is decreasing, the probability on who is the $n^{t h}$ up is "spread out". But then, $z$-independence makes it likely that one also over or undershoots by 1 . And, given that the next player is likely to hit, there must rarely be any more hits in the remaining population. Running through the players in reverse order and counting downs, when one hits $n-1$ downs, the next one must almost certainly play down, and then there must almost never be any more downs. Since both of these are true at once, in aggregate, the first $n$ bidders almost always bid up and the remaining down. Hence, $\operatorname{Pr}(I \subseteq c g) \rightarrow 1$.

The proof is long: $c g$ can move around, sometimes including one interval and sometimes another, players might bid not only above or below any given $I$, but sometimes within it, and one must be careful not to double count the ways in which a population "one player away" from creating a long $c g$ might end up creating a long supporting gap.

\subsection{Efficiency}

We are now ready for our main theorem: 
Theorem 1 All non-trivial equilibria of the single unit demand/supply double auction are asymptotically efficient. Uniformly across non-trivial equilibria, efficiency losses go to zero faster than $1 / n^{1-\alpha}$ for any given $\alpha>0$. The fraction of expected surplus lost compared to a Walrasian market thus shrinks as $1 / n^{2-\alpha}$.

For intuition, note that in Section 4.3 we showed that the efficiency loss from failing to trade objects between sellers with value below $\underline{c g}$ and buyers with values above $\overline{c g}$ is small (of order $1 / n$ ). So, the only efficiency losses to worry about are from pairs of buyers and sellers both having value in $c g$. The loss from missing such a trade is at most $|c g|$. And, using NAA, the number of such buyers and sellers is like $|c g| n$. So, the deadweight loss triangle from too little trade has area $|c g|^{2} n$. But, from Lemma $9, \operatorname{Pr}(|c g| \geq x) \leq \frac{1}{n^{2-\alpha} x^{2}}$, and so the expected loss here is like $1 / n$ as well. Finally, from NAG, expected feasible surplus grows like $n$, and so proportional losses are like $1 / n^{2}$. A formal accounting of efficiency losses is subsumed by the proof of the multiple unit case, and so omitted in the appendix.

\subsection{Asymptotic Uniqueness of Equilibrium}

In the space of allocations, all non-trivial equilibria converge to the Walrasian outcome. Over "relevant" ranges bids must thus converge to true values. So, if in the limit, the Walrasian price is either $p_{1}$ or $p_{2}>p_{1}$, then, players with value near $p_{1}$ or $p_{2}$ must bid close to value. But it is difficult to show that a player, e.g., with value well above $p_{2}$ must bid near value. A rate of convergence result for bids is thus cumbersome. Intuitively, over relevant ranges convergence should be order $1 / n$.

\section{Multiple-Unit Demands and Supplies}

Assume now that each player has demand or supply for at most $m$ units, for some fixed $m$. For buyers, let $v_{i h}, h \in\{1, \ldots, m\}$, be $i$ 's incremental value for unit $h{ }^{16}$ For sellers, let $v_{i h}$ be the incremental cost of unit $h$. We assume $v_{i h}$ is non-increasing in $h$ for buyers and non-decreasing for sellers. Bids are (non-increasing for buyers, non-decreasing for sellers) $m$-vectors. JS applies to show existence of equilibria in this setting, subject to the same strengthenings as before.

We assume the following version of NAG.

Assumption 5 (No Asymptotic Gaps*) There is $w>0$ such that for all $n$, and for all intervals $I \subseteq(0,1)$ of length $1 / n$ or greater,

$$
\sum_{i \in N_{B}} P_{i}\left[v_{i 1} \in I\right] \geq w n|I|
$$

\footnotetext{
${ }^{16}$ As before, we include atoms for buyers at 0 and sellers at 1 . So, this does not imply that buyers have positive value for all $m$ units or that sellers are want or are able to sell $m$ units.
} 
and

$$
\sum_{i \in N_{S}} P_{i}\left[v_{i 1} \in I\right] \geq w n|I|
$$

That is, when $n$ is large, there are many buyers whose highest value might fall in any given interval, and many sellers whose lowest cost might fall into any given interval. ${ }^{17}$

As before, $z$-independence applies only across players, and does not restrict the relationship of the different values of any given player. NAA is assumed to apply to all values, not just the first. So, not too many $v_{i h}$ fall in any given interval.

Theorem 2 With $N A G^{*}$, Theorem 1 continues to hold even with multiple-unit demands and supplies.

Most of the incentive arguments rely only on the highest value unit of demand for buyers and lowest cost unit for sellers. The proof proceeds in two steps. Define $\overline{u g}$ as the $m^{t h}$ bid up from $\overline{c g}$, and $u g$ as $(\overline{c g}, \overline{u g})$. In the appendix, we show that Lemma 7 continues to hold for this definition of $u g$. The modification to the intuition is very small: when $u g$ is long, there are many buyers with highest value in the top half of $u g$. But, only $m$ of them can be winning a first object. Given this, Lemma 9 is easily extended as well. Instead of sorting players into those who play "up" and "down", sort them into those who make 0 up bids, 1 up bid, etc. This is notationally intensive but straightforward and hence omitted.

Finally, we must show that since $|u g|,|c g|$ and $|l g|$ shrink quickly, inefficiency in the market disappears as $1 / n$. A proof of this is in the appendix. To see the issues involved, note that for the single unit case (and for the first unit of demand in the multiple unit case), a buyer's impact on the price is small for two reasons. First, he is unlikely to be pivotal. Second, even if he is pivotal, he doesn't affect the price much, since the next bid up is likely to be close. We exploit both of these forces in showing Lemma 7 and Lemma 9 and their adaptations here.

For units of demand after their first, many buyers can simultaneously be in the position that in raising bids other than their first, they pay more for units they were already winning. To get around this, consider the deviation to honest bidding. In any given realization, let $x$ be $\overline{u g}-\underline{c g}$. This is the maximum impact of $i$ raising his $m$ bids on price. If $v_{i h}<\underline{c g}$, then the deviation is irrelevant.

If $\underline{c g} \leq v_{i h} \leq \underline{c g}+2 m x$, then $i$ may not benefit very much from any new unit won by raising $b_{i h}$, and may hurt himself by raising the price by as much as $x$ on each of $m-1$ units already being won. But, critically, because of NAA, the number of $v_{i h}$ in $(\underline{c g}, \underline{c g}+2 m x)$ is only like $n x$ (as always, ignoring constants). So, the expected cost to bidders from this case is like $E\left(n x^{2}\right)$. But, the modified versions of Lemma 7 and Lemma 9 give that $E\left(n x^{2}\right)$ is like $1 / n$.

\footnotetext{
${ }^{17}$ There are less restrictive ways in which one might generalize NAG. For example, if each buyer's first value is uniform $[3,4]$, and their second value is uniform $\left[0, v_{i 1}\right]$ then there are many buyer values in each range. An example in Section 5.1 of Swinkels (2001), suggests that this is not strong enough to gaurantee efficiency.
} 
And, the expected efficiency loss from such players not winning also falls like $1 / n$.

Consider objects with $v_{i h}$ above $\underline{c g}+2 m x$ where $i$ is already winning an $h^{t h}$ object. As before, only one of the associated bids can be $\overline{c g}$. So, the sum of costs in terms of raising these bids is at most $x$. And, $E(x) \leq 1 / n$ as well.

The remaining objects have $v_{i h}$ above $\underline{c g}+2 m x$ but are not winning. But, then the deviation to $v$ wins an extra object at price at most $\underline{c g}+x$, and raises the price by at most $x$ on $m-1$ units, for a net profit of $v_{i h}-c g-m x$. The efficiency loss from $i$ not winning object $h$ is at most $v_{i h}-\underline{c g}$, which, given that $v_{i h}-\underline{c g}>2 m x$, is at most twice $v_{i h}-\underline{c g}-x$. So, on these objects, bidder's profits from the deviation are at least half of the efficiency loss on these units. Since costs from raising bids on other units are insignificant, it follows that the efficiency loss on these units is small since otherwise bidders will in aggregate have a profitable deviation. As the efficiency loss on other units is also small, we are done.

\section{Extensions}

\subsection{One-sided Uniform-price Auctions}

Swinkels (2001) considers large one-sided auctions with independent values and a little bit of "noise." An example is if there is a small independent probability that each player sleeps through the auction. In the uniform price case, it is shown that with the noise, the impact that any given player has on the price grows small in expectation. But then, since "honest" bidding has a small effect on the price paid, it must also have little benefit in winning extra objects. This implies asymptotic efficiency (without a rate of convergence).

An easy extension to the arguments here shows that a one sided uniform price auction with $z$-independent values converges to efficiency at rate $1 / n^{2-\alpha}$, even without noise. This paper thus significantly generalizes Swinkels (2001) for the uniform price case. The key is that here we think of "cost" as the impact on price in circumstances where the player affecting the price cares. This is a simpler object to bound, allowing both the greater generality, and fast convergence. ${ }^{18}$

\subsection{Weaker Information Assumptions}

We can weaken the information assumptions considerably. There is no problem if most players have considerably more knowledge about each other's values than $z$-independence allows. What counts (for convergence, rates are more delicate) is that from the point of view of a non-vanishing fraction of players, there are "lots" of players who he cannot predict precisely, and that NAG applies to this set of players.

\footnotetext{
${ }^{18}$ The stronger notion of vanishing impact is needed to prove results for discriminatory auctions, which are also analyzed in that paper.
} 


\subsection{Non-private Values}

We can also weaken the assumption of private values somewhat. Assume that an $\varepsilon$ fraction of the players have private values, and the remainder some sort of common. The arguments above show that over relevant ranges, the players with private values bid close to value. NAG implies that their bids are then closely packed almost surely. Thus, the impact of bids on price disappears for all players. But then, common value types should bid nearly "honestly" (their bid should nearly equal the expected value of object conditional on being pivotal). Working out such a model is left to future work.

\section{REFERENCES}

Al-Najjar, Nabil I., and Rann Smorodinsky (1997) "Pivotal Players and the Characterization of Influence," Journal of Economic Theory 92 (2), 318-342.

Chatterjee, Kalin, and William Samuelson (1983) "Bargaining Under Asymmetric Information," Operations Research, 31 835-851.

Fudenberg, Drew, Markus Mobius, and Adam Szeidl (2003) "Existence of Equilibrium in Large Double Auctions," mimeo, Harvard University.

Gresik, Tom, and Mark Satterthwaite (1989) "The Rate of Which a Simple Market Becomes Efficient as the Number of Traders Increases: An Asymptotic Result for Optimal Trading Mechanisms," Journal of Economic Theory, 48 304-332.

Jackson, Matthew O., Leo Simon, Jeroen M. Swinkels, and William Zame (2002) "Communication and Equilibrium in Discontinuous Games of Incomplete Information," Econometrica 70 (5), 1711-1740.

Mailath, George and Andrew Postlewaite (1990) "Asymmetric Information Bargaining Problems with Many Agents," Review of Economic Studies, 57 351-367.

Jackson, Matthew O. and Jeroen M. Swinkels (2001) "Existence of Equilibrium in Single and Double Private Value Auctions," mimeo John M. Olin School of Business.

McLean, Richard, and Andrew Postlewaite (2002) "Informational Size and Incentive Compatibility," Econometrica 70, 2421-2454.

Milgrom, Paul, (1979) "A Convergence Theorem for Competitive Bidding with Differential Information," Econometrica 47, 670-688.

Milgrom, Paul and Robert J. Weber (1985) "Distributional Strategies for Games with Incomplete Information," Mathematics of Operations Research, 10, 619-632.

Perry, Motty, and Philip J. Reny (2003) "Toward a Strategic Foundation for Rational Expectations Equilibrium," mimeo, University of Chicago.

Peters, Mike and Sergei Severinov (2002) "Internet Auctions with Many Traders," Mimeo, UBC. 
Pesendorfer, Wolfgang and Jeroen M. Swinkels (1997) "The Loser's Curse and Information Aggregation in Common Value Auctions," Econometrica 65, 1247-1282.

Postlewaite, Andrew, and David Schmeidler (1986) "Implementation in Differential Information Economies," Journal of Economic Theory 39, 14-33.

Rustichini, Aldo, Mark A. Satterthwaite and Steven R. Williams (1994) "Convergence to Efficiency in a Simple Market with Incomplete Information," Econometrica 62 (1), 1041-1063.

Satterthwaite, Mark A. and Steven R. Williams (1989) "The Rate of Convergence to Efficiency in the Buyer's Bid Double Auction as the Market Becomes Large," Review of Economic Studies 56, 477-498.

Satterthwaite, Mark A. and Steven R. Williams (2002) "The Optimality of a Simple Market Mechanism," Econometrica 70 (5), 1841-1864.

Shiryaev, Albert N. (1996) Probability, Springer-Verlag, Berlin, Heidelberg.

Swinkels, Jeroen M. (2001) "Efficiency of Large Private Value Auctions," Econometrica 69 (1), 37-68.

Wilson, Robert (1977) "A Bidding Model of Perfect Competition," Review of Economic Studies 66, 511-518.

Wilson, Robert (1985) "Incentive Efficiency of Double Auctions," Econometrica 53, 1101-1115.

\section{Appendix}

\subsection{Proofs for Section 3.2}

Proof of Lemma $1 \mathrm{Wlog}$, let $K=\{1,2, \ldots,|K|\}$. Let $P_{K}$ and $P_{N \backslash K}$ be the marginals of $P$ on $K$ and $N \backslash K$ respectively, and let $P_{K \times N \backslash K}$ be the associated product measure. Fix a rectangular event $F_{K}=F_{1} \cap F_{2} \cap \ldots \cap F_{|K|}$, where $F_{i}$ only involves $v_{i}$.

$$
\begin{aligned}
\operatorname{Pr}\left(F_{K} \mid F_{N \backslash K}\right) & =\prod_{i=1}^{|K|} \operatorname{Pr}\left(F_{i} \mid F_{i+1} \cap \ldots \cap F_{|K|} \cap F_{N \backslash K}\right) \\
& \leq z^{-|K|} \prod_{i=1}^{|K|} \operatorname{Pr}\left(F_{i} \mid F_{i+1} \cap \ldots \cap F_{|K|}\right) \text { (using } z-\text { independence) } \\
& =z^{-|K|} \operatorname{Pr}\left(F_{K}\right)=z^{-|K|} P_{K}\left(F_{K}\right)
\end{aligned}
$$

Analogously,

$$
\operatorname{Pr}\left(F_{K} \mid F_{N \backslash K}\right) \geq z^{|K|} P_{K}\left(F_{K}\right) .
$$

These inequalities extend to any $F_{K}$ in the product $\sigma$-algebra, as such a set is the limit of a countable union of rectangles. Thus

$$
z^{-|K|} \operatorname{Pr}\left(F_{K}\right) \operatorname{Pr}\left(F_{N \backslash K}\right) \geq \operatorname{Pr}\left(F_{K} \cap F_{N \backslash K}\right) \geq z^{|K|} \operatorname{Pr}\left(F_{K}\right) \operatorname{Pr}\left(F_{N \backslash K}\right)
$$


or equivalently

$$
z^{-|K|} P_{K \times N \backslash K} \geq P \geq z^{|K|} P_{K \times N \backslash K}
$$

Let $F_{K}$ and $F_{N \backslash K}$ be events about values and bids. Then

$$
\begin{aligned}
\operatorname{Pr}\left(F_{K} \cap F_{N \backslash K}\right) & =\int_{[0,1]|N|} \operatorname{Pr}\left(F_{K} \mid v_{K}\right) \operatorname{Pr}\left(F_{N \backslash K} \mid v_{N \backslash K}\right) d P \\
& \leq z^{-|K|} \int_{[0,1]^{|N|}} \operatorname{Pr}\left(F_{K} \mid v_{K}\right) \operatorname{Pr}\left(F_{N \backslash K} \mid v_{N \backslash K}\right) d P_{K \times N \backslash K} \\
& =z^{-|K|} \int_{[0,1]^{|K|}} \operatorname{Pr}\left(F_{K} \mid v_{K}\right) d P_{K} \int_{[0,1]^{|N|-|K|}} \operatorname{Pr}\left(F_{N \backslash K} \mid v_{N \backslash K}\right) d P_{N \backslash K} \\
& =z^{-|K|} \operatorname{Pr}\left(F_{K}\right) \operatorname{Pr}\left(F_{N \backslash K}\right) .
\end{aligned}
$$

The first integral is defined by the players' distributional strategies. The second line uses (10). The third line applies Fubini's Theorem. The final line integrates. Similarly $\operatorname{Pr}\left(F_{K} \cap F_{N \backslash K}\right) \geq z^{|K|} \operatorname{Pr}\left(F_{K}\right) \operatorname{Pr}\left(F_{N \backslash K}\right)$ so (9) holds for all events.

Similarly, for rectangular events $F_{N \backslash K}$,

$$
z^{-|N \backslash K|} \operatorname{Pr}\left(F_{K}\right) \operatorname{Pr}\left(F_{N \backslash K}\right) \geq \operatorname{Pr}\left(F_{K} \cap F_{N \backslash K}\right) \geq z^{|N \backslash K|} \operatorname{Pr}\left(F_{K}\right) \operatorname{Pr}\left(F_{N \backslash K}\right)
$$

Combining,

$$
z^{-a} \operatorname{Pr}\left(F_{K}\right) \operatorname{Pr}\left(F_{N \backslash K}\right) \geq \operatorname{Pr}\left(F_{K} \cap F_{N \backslash K}\right) \geq z^{a} \operatorname{Pr}\left(F_{K}\right) \operatorname{Pr}\left(F_{N \backslash K}\right)
$$

Dividing through by $\operatorname{Pr}\left(F_{N \backslash K}\right)$ gives (2).

Let $X_{K}$ be a step function with values $x^{\alpha}$ on a finite partition $\left\{F^{\alpha}\right\}_{\alpha \in A}$ where each $F^{\alpha}$ is an event on bids and values in $K$. By the definition of conditional expectation $E\left(X_{K} \mid F_{N \backslash K}\right)=\sum_{\alpha \in A} x^{\alpha} \operatorname{Pr}\left(F^{\alpha} \mid F_{N \backslash K}\right)$. Thus by (2)

$$
E\left(X_{K} \mid F_{N \backslash K}\right) \leq z^{-a} \sum_{\alpha \in A} x^{\alpha} \operatorname{Pr}\left(F^{\alpha}\right)=z^{-a} E\left(X_{K}\right) .
$$

Analogously, $E\left(X_{K} \mid F_{K \backslash N}\right) \geq z^{a} E\left(X_{K}\right)$. As an arbitrary $X_{K}$ is the limit of such step functions, (3) follows.

\subsection{Proofs for Section 3.3}

Proof of Lemma $2 \mathrm{~W} \log$, let $K=\{1,2, \ldots, \kappa\}$. Define the Bernoulli process with $\kappa$ independent trials with success probability $\bar{p}_{i}$ in trial $i$. Let $x_{i} \in\{0,1\}$ be the outcome of trial $i$ and let $X^{k}=\sum_{i=1}^{k} x_{i}$. We claim that $X_{K} \equiv X^{\kappa}$ FOSD $Q_{K}$ given $F_{N \backslash K}$. The proof is inductive. Let $Q^{k}$ be the number of $F_{1}, \ldots, F_{k-1}$ that occur. Trivially, $X^{0}$ FOSD $Q^{0}$, since both are identically 0. Suppose $X^{k-1}$ 
FOSD $Q^{k-1}$ given $F_{N \backslash K}$. Then, for $r \in\{0, \ldots, k\}$,

$$
\begin{aligned}
\operatorname{Pr}\left(Q^{k} \leq r \mid F_{N \backslash K}\right)= & \operatorname{Pr}\left(Q^{k-1}<r \mid F_{N \backslash K}\right) \\
& \quad+\operatorname{Pr}\left(F_{k}^{c} \mid\left\{Q^{k-1}=r\right\} \cap F_{N \backslash K}\right) \operatorname{Pr}\left(Q^{k-1}=r \mid F_{N \backslash K}\right) \\
\geq & \operatorname{Pr}\left(X^{k-1}<r \mid F_{N \backslash K}\right)+\left(1-\bar{p}_{k}\right) \operatorname{Pr}\left(X^{k-1}=r \mid F_{N \backslash K}\right) \\
= & \operatorname{Pr}\left(X_{K} \leq r\right) .
\end{aligned}
$$

The inequality uses $z$-independence and the inductive hypothesis.

Similarly, if $Y_{K}$ is the number of successes in a Bernoulli process with success probabilities $\underline{p}_{i}$ then given $F_{N \backslash K}, Q_{K}$ FOSD $Y_{K}$.

We want a large-deviations inequality for the bounding Bernoulli processes. As $X_{K}$ is a sum of non-identical independent Bernoulli trials, a slight alteration to the usual proof of Cramér's Theorem (e.g., Shirayev (1996) p.68) is necessary. Let $\pi=\frac{1}{\kappa} \sum_{i} \bar{p}_{i}$. Then, for any $\lambda>0$,

$$
\operatorname{Pr}\left(\frac{X_{K}}{\kappa \pi}>\phi\right)=\operatorname{Pr}\left(e^{\lambda X_{K} / \kappa \pi} \geq e^{\lambda \phi}\right) \leq \frac{E\left(e^{\lambda X_{K} / \kappa \pi}\right)}{e^{\lambda \phi}}
$$

by Markov's inequality. Note also that $\operatorname{Pr}\left(\frac{X_{K}}{\kappa \pi}>\phi\right)=0$ trivially when $\pi \phi \geq 1$.

Now, as $X_{K}$ is a sum of independent random variables

$$
\begin{aligned}
E e^{\lambda X_{K} / \kappa \pi} & =\prod_{i \in K}\left(1-\bar{p}_{i}+\bar{p}_{i} e^{\lambda / \kappa \pi}\right) \\
& =\exp \left(\log \prod_{i \in K}\left(1-\bar{p}_{i}+\bar{p}_{i} e^{\lambda / \kappa \pi}\right)\right) \\
& =\exp \left(\sum_{i \in K} \log \left(1-\bar{p}_{i}+\bar{p}_{i} e^{\lambda / \kappa \pi}\right)\right) \\
& \leq \exp \left(\kappa \log \left(1-\pi+\pi e^{\lambda / \kappa \pi}\right)\right)
\end{aligned}
$$

since $\log \left(1-x+x e^{\lambda / \kappa \pi}\right)$ is concave in $x$.

Thus,

$$
\begin{aligned}
\operatorname{Pr}\left(\frac{X_{K}}{\kappa \pi}>\phi\right) & \leq \exp \left[-\lambda \phi+\kappa \log \left(1-\pi+\pi e^{\lambda / \kappa \pi}\right)\right] \\
& =\exp \left[-\kappa\left\{\frac{\lambda}{\kappa \pi} \phi \pi-\kappa \log \left(1-\pi+\pi e^{\lambda / \kappa \pi}\right)\right\}\right] \\
& =\exp \left[-\kappa\left\{s \phi \pi-\log \left(1-\pi+\pi e^{s}\right)\right\}\right]
\end{aligned}
$$

where $s \equiv \frac{\lambda}{\kappa \pi}$. Given that $\lambda>0$ was arbitrary, this holds for all $s>0$, and so 
in particular for $s=\log \left(\frac{(1-\pi) \phi}{1-\pi \phi}\right)$ (this is positive, because $\pi \phi<1$ ), yielding

$$
\begin{aligned}
\operatorname{Pr}\left(\frac{X_{K}}{\kappa \pi}>\phi\right) & \leq \exp \left[-\kappa\left\{\log \left(\frac{(1-\pi) \phi}{1-\pi \phi}\right) \phi \pi-\log \left(1-\pi+\pi e^{\log \left(\frac{(1-\pi) \phi}{1-\pi \phi}\right)}\right)\right\}\right] \\
& =\exp \left[-\kappa\left\{\phi \pi \log \phi+(1-\phi \pi) \log \left(\frac{1-\phi \pi}{1-\pi}\right)\right\}\right]
\end{aligned}
$$

As $\log x \geq(x-1) / x$ the second term in the braces is at least $\pi(1-\phi)$. Thus,

$$
\operatorname{Pr}\left(\frac{X_{K}}{\kappa \pi}>\phi\right) \leq \exp [-\kappa \pi(\phi \log \phi+1-\phi)]
$$

Choosing $\phi=3$,

$$
\operatorname{Pr}\left(X_{K}>3 \kappa \pi\right) \leq e^{-\kappa \pi(\log 27-2)} \leq e^{-\kappa \pi} .
$$

Note that $\frac{1}{z} E\left(Q_{K}\right) \geq \sum_{i \in K} \bar{p}_{i}=\kappa \pi$, and hence $\operatorname{Pr}\left(X_{K}>3 \kappa \pi\right) \geq \operatorname{Pr}\left(X_{K}>\frac{3}{z} E\left(Q_{K}\right)\right)$. And, $\sum_{i \in K} \bar{p}_{i} \geq E\left(Q_{K}\right)$, and so $e^{-\kappa \pi} \leq e^{-E\left(Q_{K}\right)}$. Finally, $X_{K}$ stochastically dominates $Q_{K}$. Taken together with (16), this implies

$$
\operatorname{Pr}\left(Q_{K}>\frac{E\left(Q_{K}\right)}{z} \mid F\right) \leq e^{-E\left(Q_{K}\right)}
$$

giving (5).

The proof for $Y_{K}$ is similar: Define $\pi=\sum_{i \in K} p_{i}$. Then, for any $\lambda<0$, and $0<\phi<1$

$$
\operatorname{Pr}\left(\frac{Y_{K}}{\kappa \pi}<\phi\right)=\operatorname{Pr}\left(e^{\lambda X_{K} / \kappa \pi} \geq e^{\lambda \phi}\right) \leq \frac{E\left(e^{\lambda X_{K} / \kappa \pi}\right)}{e^{\lambda \phi}} .
$$

The derivation of (13) and (14) is then as before, replacing $\bar{p}_{i}$ by $\underline{p}_{i}$ and $\operatorname{Pr}\left(\frac{X_{K}}{\kappa \pi}>\phi\right)$ by $\operatorname{Pr}\left(\frac{Y_{K}}{\kappa \pi}<\phi\right)$. Note in particular that since $\lambda<0, s \equiv \frac{\lambda}{\kappa \pi}$ can once again take on any positive value. Setting $s=\log \left(\frac{(1-\pi) \phi}{1-\pi \phi}\right)$ is once again valid, as $\phi<1$, hence we arrive at the analog to (15):

$$
\operatorname{Pr}\left(\frac{Y_{K}}{\kappa \pi}<\phi\right) \leq \exp [-\kappa \pi(\phi \log \phi+1-\phi)] .
$$

Note that $\sum_{i \in K} p_{i} \geq z E\left(Q_{K}\right)$, so that $\operatorname{Pr}\left(Y_{K}<\phi z E\left(Q_{K}\right)\right) \leq \operatorname{Pr}\left(Y_{K}<\phi \kappa \pi\right)$ and $\exp [-\kappa \pi(\phi \log \phi+1-\phi)] \leq \exp \left[-z E\left(Q_{K}\right)(\phi \log \phi+1-\phi)\right]$. So,

$$
\operatorname{Pr}\left(Q_{K}<\phi z E\left(Q_{K}\right)\right) \leq e^{-z E\left(Q_{K}\right)(\phi \log \phi+1-\phi)} .
$$

Since $\frac{1}{3} \log \frac{1}{3}+1-\frac{1}{3}>0.3$, (4) follows

Proof of Corollary 1 Note that $\operatorname{Pr}\left(Q_{K}=0 \mid F_{N \backslash K}\right) \leq \operatorname{Pr}\left(Q_{K} \leq \phi E\left(Q_{K}\right)\right)$ for any $\phi>0$. Equation 18 then gives

$$
\begin{aligned}
\operatorname{Pr}\left(Q_{K}=0 \mid F_{N \backslash K}\right) & \leq \operatorname{Pr}\left(Q_{K} \leq \frac{z}{3} E\left(Q_{K}\right)\right) \\
& \leq e^{-z E\left(Q_{K}\right)(\phi \log \phi+1-\phi)} \\
& \leq e^{-z \operatorname{Pr}\left(Q_{K} \geq 1\right)(\phi \log \phi+1-\phi)}
\end{aligned}
$$


since $E\left(Q_{K}\right) \geq \operatorname{Pr}\left(Q_{K} \geq 1\right)$. As this holds for $\phi$ arbitrarily close to 0 ,

$$
\begin{aligned}
\operatorname{Pr}\left(Q_{K} \geq 1 \mid F_{N \backslash K}\right) & \geq 1-e^{-z \operatorname{Pr}\left(Q_{K} \geq 1\right)} \\
& =\frac{1-e^{-z \operatorname{Pr}\left(Q_{K} \geq 1\right)}}{\operatorname{Pr}\left(Q_{K} \geq 1\right)} \operatorname{Pr}\left(Q_{K} \geq 1\right)
\end{aligned}
$$

For $x \in(0,1],\left(1-e^{-z x}\right) / x$ is minimized at $x=1$.

\subsection{Proofs for Section 3.4}

Proof of Lemma 3 Partition $[0,1]$ into $k \equiv\left[n^{1-\alpha / 4}\right]$ intervals $\left\{I_{\kappa}\right\}$ of equal length (between $n^{1-\alpha / 4}$ and $2 n^{1-\alpha / 4}$ ). Let $Q_{B}\left(I_{\kappa}\right)$ be the number of buyers with values in $I_{\kappa}$. Note that

$$
W n / k=W n\left|I_{\kappa}\right| \geq E\left(Q_{B}\left(I_{\kappa}\right)\right) \geq w n\left|I_{\kappa}\right|=w n / k .
$$

Let $E_{1 \kappa} \equiv\left\{\frac{3}{z} W n / k \geq Q_{B}\left(I_{\kappa}\right) \geq \frac{z}{3} w n / k\right\}$. By Lemma 2 ,

$$
\operatorname{Pr}\left(E_{1 \kappa}\right) \geq 1-2 e^{-.3 z n / k} \geq 1-\frac{1}{n^{5}}
$$

for $n$ SL, since $n / k \rightarrow n^{\alpha / 4}$. Similarly, let $Q_{S}\left(I_{\kappa}\right)$ be the number of sellers with values in $I_{\kappa}$, and define $E_{2 \kappa}=\left\{\frac{3}{z} W n / k \geq Q_{S}(I) \geq \frac{z}{3} w n / k\right\}$. Then, $\operatorname{Pr}\left(E_{2 \kappa}\right) \geq$ $1-\frac{1}{n^{5}}$ for $n$ SL.

Then, $\mathcal{N} \equiv \cap_{\kappa}\left(E_{1 \kappa} \cap E_{2 \kappa}\right)$. As this involves $2 k \leq 2 n^{1-\alpha / 4}$ events,

$$
\begin{aligned}
\operatorname{Pr}(\mathcal{N}) & \geq 1-2 \frac{n^{1-\alpha / 4}}{n^{5}} \\
& \geq 1-1 / n^{4}
\end{aligned}
$$

for $n$ SL.

Finally, note that for $n \mathrm{SL}$, any interval $I$ of length at least $\frac{1}{n^{1-\alpha / 3}}$ contains at least $k|I| / n-2 \geq k|I| / 2 n$ elements of $\left\{I_{\kappa}\right\}$. So, in a normal realization,

$$
Q_{B}(I) \geq \frac{k|I|}{2 n} \frac{z}{3} w n / k=\frac{z w}{6}|I| .
$$

Similarly, $I$ intersects with at most $2 k|I| / n$ elements of $\left\{I_{\kappa}\right\}$ and so $Q_{B}(I) \leq$ ${ }_{z}^{6} W|I|$. The argument for $Q_{S}(I)$ is analogous.

\section{Proofs for Section 4.2}

Proof of Lemma 5 If $\frac{E(V \mid T)}{n} \nrightarrow 0$, then along a subsequence, $\operatorname{Pr}(V>\gamma n \mid T)>$ $\gamma$ for some $\gamma$. Given $\{V>\gamma n\}$, if one selects $\frac{\gamma n}{2}$ of the buyers at random, the probability that none trades is at most $(1-\gamma)^{\frac{\gamma n}{2}} \leq 1 / 8$ for $n \mathrm{SL}$, and so there is a $7 / 8$ probability of at least one trader. Since this is true in expectation, it must be true for some particular set $G_{B}$ of $\frac{\gamma n}{2}$ buyers. Similarly, there is a set $G_{S}$ 
of $\gamma n / 2$ sellers such that conditional on $\{V>\gamma n\}$ at least one is a trader with probability $7 / 8$. Let $G \equiv G_{S} \cup G_{B}$, and let $T_{G}$ be the event that at least one buyer and one seller in $G$ trades. Then, $\operatorname{Pr}\left(T_{G} \mid\{V>\gamma n\}\right) \geq 1-2(1 / 8)=3 / 4$. So, $\operatorname{Pr}\left(T_{G} \cap\{V>\gamma n\}\right) \geq 3 / 4 \operatorname{Pr}(V>\gamma n)$. As $T_{G} \subseteq T$,

$$
\begin{aligned}
\operatorname{Pr}\left(\{V>\gamma n\} \mid T_{G}\right) & =\frac{\operatorname{Pr}\left(T_{G} \cap\{V>\gamma n\}\right)}{\operatorname{Pr}\left(T_{G}\right)} \\
& \geq \frac{3 / 4 \operatorname{Pr}(V>\gamma n)}{\operatorname{Pr}(T)} \\
& \geq 3 \gamma / 4 .
\end{aligned}
$$

Since $G$ has only $\frac{\gamma n}{2}$ buyers or sellers, $T_{G} \cap\{V>\gamma n\}$ implies that there are at least $\frac{\gamma n}{2}$ buyers and sellers trading in $N \backslash G$. Let $X$ be this event. So, $\operatorname{Pr}\left(X \mid T_{G}\right) \geq 3 \gamma / 4$.

Let $p^{*}$ be such that $\operatorname{Pr}\left(p \geq p^{*} \mid X \cap T_{G}\right) \geq \frac{1}{2}$ and $\operatorname{Pr}\left(p \leq p^{*} \mid X \cap T_{G}\right) \geq \frac{1}{2}$. Let $Q_{S}$ be the number of sellers in $N \backslash G$ with $b_{i} \leq p^{*}$ and $Q_{B}$ the number of buyers in $N \backslash G$ with $b_{i} \geq p^{*}$. Then,

$$
E\left(Q_{S} \mid T_{G}\right) \geq \operatorname{Pr}\left(X \cap\left\{p \leq p^{*}\right\} \mid T_{G}\right) \frac{\gamma n}{2} \geq \frac{1}{2} \operatorname{Pr}\left(X \mid T_{G}\right) \frac{\gamma n}{2} \geq 3 \gamma^{2} n / 16,
$$

and so

$E\left(Q_{S}\right)=\sum_{i \in N_{S} \backslash G} \operatorname{Pr}\left(b_{i} \leq p^{*}\right) \geq z \sum_{i \in N_{S} \backslash G} \operatorname{Pr}\left(b_{i} \leq p^{*} \mid T_{G}\right)=z E\left(Q_{S} \mid T_{G}\right)=3 z \gamma^{2} n / 16$.

Thus by Lemma $2 \operatorname{Pr}\left(Q_{S}=0\right) \rightarrow 0$. Similarly $\operatorname{Pr}\left(Q_{B}=0\right) \rightarrow 0$. But then, $\operatorname{Pr}(T) \rightarrow 1$.

Proof of Proposition 1 Fix $\mathcal{A}^{n}$ and a non-trivial equilibrium. Let $\phi_{B} \equiv$ $\max _{N_{B}} b_{i}$ be the highest buy bid submitted and let $\phi_{S} \equiv \min _{N_{S}} b_{i}$ be the lowest sell bid. Note that $\operatorname{Pr}\left(\phi_{B} \geq x\right)$ is decreasing and continuous from the left. Similarly, $\operatorname{Pr}\left(\phi_{S} \leq x\right)$ is increasing and continuous from the right. Let $v^{*} \in[0,1]$ have the property that for all $x \in\left[0, v^{*}\right), \operatorname{Pr}\left(\phi_{B} \geq x\right) \geq \operatorname{Pr}\left(\phi_{S} \geq x\right)$, while for all $x \in\left(v^{*}, 1\right], \operatorname{Pr}\left(\phi_{B} \geq x\right) \leq \operatorname{Pr}\left(\phi_{S} \geq x\right)$. Let

$$
\delta \equiv \min \left\{\operatorname{Pr}\left(\phi_{B} \geq v^{*}\right), \operatorname{Pr}\left(\phi_{S} \leq v^{*}\right)\right\} .
$$

Note that $\operatorname{Pr}\left(\phi_{B}>v^{*}\right) \leq \delta$. This is trivial if $\operatorname{Pr}\left(\phi_{B} \geq v^{*}\right)=\delta$. If $\operatorname{Pr}\left(\phi_{B} \geq\right.$ $\left.v^{*}\right)>\delta$, then $\operatorname{Pr}\left(\phi_{S} \geq v^{*}\right)=\delta$. But then since $\operatorname{Pr}\left(\phi_{S} \leq x\right)$ is continuous from the right,

$$
\begin{aligned}
\operatorname{Pr}\left(\phi_{B}>v^{*}\right) & =\lim _{v \downarrow v^{*}} \operatorname{Pr}\left(\phi_{B} \geq v\right) \\
& \leq \lim _{v \downarrow v^{*}} \operatorname{Pr}\left(\phi_{S} \geq v\right) \\
& =\operatorname{Pr}\left(\phi_{S} \geq v\right)=\delta .
\end{aligned}
$$


Analogously, $\operatorname{Pr}\left(\phi_{S}<v^{*}\right) \leq \delta$.

Assume that $\operatorname{Pr}\left(\phi_{S} \leq v^{*}\right)=\delta$. Then, $\operatorname{Pr}\left(T \cap\left\{p \leq v^{*}\right\}\right) \leq \operatorname{Pr}\left(\phi_{S} \leq v^{*}\right)=\delta$, while $\operatorname{Pr}\left(T \cap\left\{p>v^{*}\right\}\right) \leq \operatorname{Pr}\left(\phi_{B}>v^{*}\right) \leq \delta$. Similarly, if $\operatorname{Pr}\left(\phi_{B} \geq v^{*}\right)=\delta$, then, $\operatorname{Pr}\left(T \cap\left\{p<v^{*}\right\}\right) \leq \operatorname{Pr}\left(\phi_{S}<v^{*}\right) \leq \delta$, while $\operatorname{Pr}\left(T \cap\left\{p \geq v^{*}\right\}\right) \leq \operatorname{Pr}\left(\phi_{B} \geq\right.$ $\left.v^{*}\right)=\delta$. So $\operatorname{Pr}(T) \leq 2 \delta$.

Now, $\left\{\phi_{S} \leq v^{*}\right\}=\cup_{i \in N_{S}}\left\{b_{i} \leq v^{*}\right\}$. Hence, by Corrolary 1,

$$
\begin{aligned}
\operatorname{Pr}\left(\phi_{S} \leq v^{*} \mid F_{N_{B}}\right) & \geq\left(1-e^{-z}\right) \operatorname{Pr}\left(\phi_{S} \leq v^{*}\right) \\
& \geq\left(1-e^{-z}\right) \delta
\end{aligned}
$$

for any $F_{N_{B}}$. So,

$$
\begin{aligned}
\operatorname{Pr}(T) & \geq \operatorname{Pr}\left(\left\{\phi_{B} \geq v^{*}\right\} \cap\left\{\phi_{S} \leq v^{*}\right\}\right) \\
& \geq \operatorname{Pr}\left(\phi_{S} \leq v^{*} \mid \phi_{B} \geq v^{*}\right) \operatorname{Pr}\left(\phi_{B} \geq v^{*}\right) \\
& \geq\left(1-e^{-z}\right) \delta^{2}
\end{aligned}
$$

Assume that $v^{*} \leq 1 / 2$. (If not, the proof below applies, mutatis mutandis, to the sellers). Fix an arbitrary buyer $i$. Let $\phi_{B}^{i} \equiv \max _{N_{B} \backslash\{i\}} b_{i}$. Now,

$$
\begin{aligned}
\operatorname{Pr}\left(\phi_{B}^{i}<2 / 3\right) & =1-\operatorname{Pr}\left(\phi_{B} \geq 2 / 3\right) \\
& \geq 1-\operatorname{Pr}\left(\phi_{B}>v^{*}\right) \\
& \geq 1-\delta .
\end{aligned}
$$

Let $J \equiv[5 / 6,1]$. By Lemma 1 ,

$$
\operatorname{Pr}\left(\phi_{B}^{i}<2 / 3 \mid v_{i} \in J\right) \geq z(1-\delta) .
$$

By (20),

$$
\operatorname{Pr}\left(\phi_{S} \leq v^{*} \mid v_{i} \in J, \phi_{B}^{i}<v^{*}\right) \geq\left(1-e^{-z}\right) \delta .
$$

Let $d_{i}$ be the deviation for $i$ that whenever $v_{i} \in J$ and the original strategy specified a bid below $v^{*}$, he bids $2 / 3$ instead. Under this strategy, he wins an object with probability at least $\operatorname{Pr}\left(\phi_{B}^{i}<2 / 3, \phi_{S} \leq v^{*}, v_{i} \in J\right)$, which by (22) and (23) is at least

$$
\operatorname{Pr}\left(v_{i} \in J\right) z(1-\delta)\left(1-e^{-z}\right) \delta,
$$

and earns at least $\frac{1}{6}$ when he does so. So,

$$
B_{i} \geq \operatorname{Pr}\left(v_{i} \in J\right) z(1-\delta)\left(1-e^{-z}\right) \delta \frac{1}{6}-\pi_{i},
$$

where $\pi_{i}$ is $i$ 's expected equilibrium profit.

Summing across buyers, and applying Lemma 4,

$$
z(1-\delta)\left(1-e^{-z}\right) \delta \frac{1}{6} \sum_{N_{B}} \operatorname{Pr}\left(v_{i} \in J\right)-\sum_{N_{B}} \pi_{i} \leq \operatorname{Pr}(T) .
$$


By A2 $\sum_{N_{B}} \operatorname{Pr}\left(v_{i} \in J\right) \geq \frac{1}{6} w n$ for $n \geq 6$. As the gains to a buyer from any given trade are at most 1 , and $V$ buyers trade,

$$
\sum_{N_{B}} \pi_{i} \leq \operatorname{Pr}(T) E(V \mid T)
$$

Substituting into (24) gives

$$
z(1-\delta)\left(1-e^{-z}\right) \delta\left(\frac{1}{6}\right)^{2} w n-2 \delta \operatorname{Pr}(T) E(V \mid T) \leq \operatorname{Pr}(T) .
$$

Using $\operatorname{Pr}(T) \leq 2 \delta$, and dividing through by $2 \delta>0$ gives

$$
\frac{1}{72} w z(1-\delta)\left(1-e^{-z}\right) n-E(V \mid T) \leq 1 .
$$

For this to hold for large $n$, either $(1-\delta)$ must be close to 0 , in which case, $\operatorname{Pr}(T) \geq\left(1-e^{-z}\right) \delta^{2} \not \rightarrow 0$ (by (21)) or, $E(V \mid T)$ must grow like $n$. But then, by Lemma $5, \operatorname{Pr}(T) \rightarrow 1$.

\subsection{Proofs for Section 4.3}

We will prove stronger results that will be useful when we turn to the multiple unit case. Fix an integer $m \geq 1$. Redefine $\overline{u g}$ as the $m^{t h}$ bid above $\overline{c g}$. As before, let $u g \equiv(\overline{c g}, \overline{u g})$. When $m=1$, we have the original case.

Proof of Lemma 6 Let $v \equiv E(|u g|)$, and let us show that for $n$ SL, $v \leq \frac{1}{n^{1-\alpha_{4}}}$. Assume this is false along a subsequence. Then

$$
\begin{aligned}
v= & \operatorname{Pr}(\mathcal{N}) E(|u g| \mid \mathcal{N})+(1-\operatorname{Pr}(\mathcal{N})) E\left(|u g| \mid \mathcal{N}^{c}\right) \\
\leq & \operatorname{Pr}\left(\mathcal{N} \cap\left\{|u g|>\frac{v}{2}\right\}\right) E\left(|u g| \mid \mathcal{N} \cap\left\{|u g|>\frac{v}{2}\right\}\right) \\
& +\operatorname{Pr}\left(\mathcal{N} \cap\left\{|u g| \leq \frac{v}{2}\right\}\right) E\left(|u g| \mid \mathcal{N} \cap\left\{|u g| \leq \frac{v}{2}\right\}\right)+\frac{1}{n^{4}} \text { (for } n \text { SL) } \\
= & \operatorname{Pr}\left(\mathcal{N} \cap\left\{|u g|>\frac{v}{2}\right\}\right) E\left(|u g| \mid \mathcal{N} \cap\left\{|u g|>\frac{v}{2}\right\}\right)+\frac{v}{2}+\frac{1}{n^{4}} .
\end{aligned}
$$

So, for $n \mathrm{SL}$

$$
\operatorname{Pr}\left(\mathcal{N} \cap\left\{|u g|>\frac{v}{2}\right\}\right) E\left(|u g| \mid \mathcal{N} \cap\left\{|u g|>\frac{v}{2}\right\}\right)>\frac{v}{3} .
$$

Consider $d_{i}\left(b_{i}, v_{i}\right)=\max \left\{b_{i}, v_{i}-\frac{\nu}{2}\right\}$. Consider $\mathcal{N} \cap\left\{|u g|>\frac{\nu}{2}\right\}$. Since $|u g|>\frac{\nu}{2}$, any buyer in the top half of $|u g|$ is a winner after $d_{i}$, and at most $m$ were winners before (since buyers bid at most $v_{i}$, and by definition, there are only $m$ bids in $[\overline{c g}, \overline{u g})$ ). By Lemma 3 (which applies since $|u g| / 2>v / 2>\frac{1}{2 n^{1-\alpha_{4}}}>\frac{1}{n^{1-\alpha / 3}}$ 
for $n \mathrm{SL}$ ), the number of new winners is at least $w^{\prime} \frac{1}{2} n|u g|-m \geq \frac{w^{\prime}}{4} n|u g|$ for $n$ SL. Each new winner earns at least $\frac{v}{2}$. So, using (25)

$$
\begin{aligned}
\sum B_{i} & \geq \frac{v}{2} \frac{w^{\prime}}{4} n E\left(|u g| \mid \mathcal{N} \cap\left\{|u g|>\frac{v}{2}\right\}\right) \operatorname{Pr}\left(\mathcal{N} \cap\left\{|u g|>\frac{v}{2}\right\}\right) \\
& \geq \frac{v}{2} \frac{w^{\prime}}{4} n \frac{v}{3} .
\end{aligned}
$$

But, by Lemma $4, \sum B_{i} \leq v$, and so

$$
\frac{v}{2} \frac{w^{\prime}}{4} n \frac{v}{3} \leq v
$$

or

$$
n v \leq \frac{24}{w^{\prime}}
$$

For $n \mathrm{SL}$, this contradicts $v \geq \frac{1}{n^{1-\alpha_{4}}}$. The argument for sellers is analogous.

Proof of Lemma 7 Assume $\operatorname{Pr}(|u g|>x)>\frac{1}{x^{2} n^{2}-\alpha_{3}}$ along a subsequence where $x>\frac{1}{n^{1-\alpha_{3} / 2}}$ (for smaller $x, \frac{1}{x^{2} n^{2-\alpha_{3}}} \geq 1$, and the claim is vacuous). Then, for $n$ $\mathrm{SL}$

$$
\operatorname{Pr}(\mathcal{N} \cap\{|u g|>x\})>\operatorname{Pr}(\{|u g|>x\})-\frac{1}{n^{4}}>\operatorname{Pr}(\{|u g|>x\}) / 2 .
$$

Consider $d_{i}\left(b_{i}, v_{i}\right)=\max \left\{b_{i}, v_{i}-\frac{x}{2}\right\}$. As before, given $\mathcal{N} \cap\{|u g|>x\}$, Lemma 3 implies that there are $w^{\prime} n x-m>w^{\prime} n x / 2$ new winners, each earning $x / 2$ (note that $x>\frac{1}{n^{1-\alpha_{3} / 2}}>\frac{1}{n^{1-a / 3}}$, so Lemma 3 does apply). So,

$$
\sum_{N_{S}} B_{i} \geq \operatorname{Pr}(|u g|>x) \frac{w^{\prime} n x^{2}}{4} .
$$

But, using Lemma 4 and Lemma $6, \sum B_{i} \leq \frac{1}{n^{1-\alpha_{4}}}$. So,

$$
\operatorname{Pr}(|u g|>x) \frac{w^{\prime} n x^{2}}{4} \leq \frac{1}{n^{1-\alpha_{4}}},
$$

Rearranging

$$
\operatorname{Pr}(|u g|>x) \leq \frac{4}{w^{\prime} n^{2-\alpha_{4}}} .
$$

For $n$ SL, this contradicts $\operatorname{Pr}(|u g|>x)>\frac{1}{x^{2} n^{2-\alpha_{3}}}$.

Proof of Lemma 8 For buyer $i$, consider $d_{i}\left(b_{i}, v_{i}\right) \equiv \max \left\{b_{i}, v_{i}-x\right\}$. If $i \in L_{B}(x)$, then $i$ wins an extra object and earns at least $x$. By Lemma 4 and Lemma 6,

$$
x E\left(l_{B}(x)\right) \leq \sum B_{i} \leq E(|u g|) \leq \frac{1}{n^{1-\alpha_{4}}}
$$

and so

$$
E\left(l_{B}(x)\right) \leq \frac{1}{x n^{1-\alpha_{4}}}
$$


establishing the first claim. Now, note that in each realization,

$$
\left.S L_{B}(1 / n)\right)=\frac{1}{n} l_{B}(1 / n)+\int_{1 / n}^{1} l_{B}(x) d x .
$$

(This is easily seen by noting that $S L_{B}(1 / n)$ is a consumer surplus calculation for demand curve $l_{B}($.$) up to demand Q=l_{B}(1 / n)$. Therefore, by Fubini's theorem

$$
\begin{aligned}
E\left(S L_{B}(1 / n)\right) & =\frac{1}{n} E\left(l_{B}(1 / n)\right)+\int_{1 / n}^{1} E\left(l_{B}(x)\right) d x \\
& \leq \frac{1}{n^{1-\alpha_{4}}}+\int_{1 / n}^{1} \frac{1}{x n^{1-\alpha_{4}}} d x \text { using (26) twice } \\
& =\frac{1}{n^{1-\alpha_{4}}}(1+\log n) \\
& \leq \frac{1}{n^{1-\alpha_{3}}}(\text { for } n \mathrm{SL})
\end{aligned}
$$

which establishes the second claim. Repeat for sellers.

\subsection{Proofs for Section 4.4}

Proof of Lemma 9 Suppose the lemma is false, so that there exists a sequence $\left\{n_{t}\right\},\left\{x_{t}\right\}$ satisfying $n_{t} \rightarrow \infty$ and $x_{t} \geq 1 / n_{t}^{1-\alpha_{2} / 2}$ such that $\operatorname{Pr}\left(|c g| \geq x_{t}\right) \geq$ $1 / n_{t}^{2-\alpha_{2}} x_{t}^{2}$ (the claim is vacuous for smaller $x_{t}$ ).

Step 1. Sparse Intervals.

Recall from the proof of Lemma 3 the partition of $[0,1]$ into $k \equiv\left[n^{1-\alpha / 4}\right]$ disjoint intervals $\left\{I_{\kappa}\right\}$ of equal length between $1 / n^{1-\alpha / 4}$ and $2 / n^{1-\alpha / 4}$. Let $M\left(I_{\kappa}\right)$ be the number of bids in $I_{\kappa}$. Let $\omega=z w^{\prime} / 24$. Say $I_{\kappa}$ is sparse if $E\left(M\left(I_{\kappa}\right)\right)<\omega n^{\alpha / 4}$. Let $X$ be the set of sparse intervals. For $\kappa \in X$, let $E_{3 \kappa} \equiv\left\{M\left(I_{\kappa}\right) \leq \frac{3}{z} \omega n^{\alpha / 4}\right\}$ be the event that there are not "too many" bids in $I_{\kappa}$. For any given $\tau \in[0,1]$ consider the process in which at step one, values and bids are drawn according to the distributional strategy $\mu$, and at stage two, each bid is randomly and independently replaced by a bid in $I_{\kappa}$ with probabilty $\tau$. Let $M_{\tau}\left(I_{\kappa}\right)$ be the random variable given the number of bids in $I_{\kappa}$ for this process. Clearly, $M_{\tau}\left(I_{\kappa}\right)$ stochastically dominates $M\left(I_{\kappa}\right)$ for any $\tau$. Choose $\tau^{*}$ such that $E\left(M_{\tau^{*}}\left(I_{\kappa}\right)\right)=\omega n^{\alpha / 4}$. Then, Lemma 2 implies that

$$
\begin{aligned}
\operatorname{Pr}\left(E_{3 \kappa}\right) & \geq \operatorname{Pr}\left(M_{\tau^{*}}\left(I_{\kappa}\right) \leq \frac{3}{z} \omega n^{\alpha / 4}\right) \\
& \geq 1-e^{-\omega n^{\alpha / 4}} \\
& \geq 1-\frac{1}{n^{5}}
\end{aligned}
$$

for $n$ SL. 
For $\kappa \notin X$, let $E_{3 \kappa} \equiv\left\{M\left(I_{\kappa}\right) \geq \frac{z}{3} \omega n^{\alpha / 4}\right\}$ be the event that there are not "too few" bids in $I_{\kappa}$. Lemma 2 implies that for $n \mathrm{SL}$

$$
\operatorname{Pr}\left(E_{3 \kappa}\right) \geq 1-e^{-z \omega n^{\alpha / 4}} \geq 1-\frac{1}{n^{5}} .
$$

Let $\mathcal{N}^{\prime} \equiv \mathcal{N} \cap\left(\cap_{\kappa} E_{3 \kappa}\right)$. Arguing as in the proof of Lemma 3, for $n \mathrm{SL}$

$$
\begin{aligned}
\operatorname{Pr}\left(\mathcal{N}^{\prime}\right) & \geq 1-3 \frac{n^{1-\alpha / 4}}{n^{5}} \\
& \geq 1-1 / n^{4} .
\end{aligned}
$$

Step 2. Sparse Regions and the Endpoints of Competitive Gaps. Assemble maximal groups of adjacent sparse intervals into sparse regions. Let $\left\{J^{\lambda}\right\}_{\lambda \in \Lambda}$ be the set of sparse regions that are longer than $\frac{x}{2}$. For $n$ SL, $\frac{z}{3} \omega n^{\alpha / 4}>1$. So, given $\mathcal{N}^{\prime}$, for all $n$ SL, each non-sparse interval contains at least 1 bid and so cg cannot contain a non-sparse interval; cg can include at most a $J^{\lambda}$ and parts of the two non-sparse intervals immediately adjacent. These two intervals, having length at most $2 / n^{1-\alpha / 4}$ become arbitrarily short compared to $x \geq 1 / n^{1-\alpha_{2} / 2}$. Hence, given $\mathcal{N}^{\prime}$, and for $n$ SL a competitive gap of length $x$ must (a) have intersection of length at least $x / 2$ with some $J^{\lambda}$, and (b) intersect at most one $J^{\lambda}$.

Let $J_{y}^{\lambda}, y \in[0,1]$ be the point a $y^{\text {th }}$ of the way up the interval $J^{\lambda}$. Our first lemma says that it is very unlikely that the competitive gap ends a long way from the end of a $J^{\lambda}$.

Lemma 10 For all $n S L$

$$
\begin{aligned}
& \operatorname{Pr}\left(\overline{c g} \in \cup_{\lambda}\left[J_{0}^{\lambda}, J_{4 / 5}^{\lambda}\right]\right) \leq \frac{1}{12 n^{2-\alpha_{2}} x^{2}}, \\
& \operatorname{Pr}\left(\underline{c g} \in \cup_{\lambda}\left[J_{1 / 5}^{\lambda}, J_{1}^{\lambda}\right]\right) \leq \frac{1}{12 n^{2-\alpha_{2}} x^{2}} .
\end{aligned}
$$

Proof Consider the event $\left\{\overline{c g} \in\left[J_{0}^{\lambda}, J_{4 / 5}^{\lambda}\right]\right\} \cap \mathcal{N}^{\prime}$ for some $\lambda \in \Lambda$. Let $y \equiv J_{1}^{\lambda}-$ $\overline{c g}$. As $\overline{c g} \in\left[J_{0}^{\lambda}, J_{4 / 5}^{\lambda}\right], y / 2 \geq x / 10 \geq 1 / n^{1-\alpha / 4}$. So, By Lemma 3 , the number of values in $\left[J_{1}^{\lambda}-y / 2, J_{1}^{\lambda}\right]$ is at least $w^{\prime} y n / 2$. On the other hand, by Step 1 , given $\mathcal{N}^{\prime}$, each $I_{\kappa} \subseteq\left[J_{1}^{\lambda}-y / 2, J_{1}^{\lambda}\right]$ includes at most $\frac{3}{z} \omega n^{\alpha / 4}=\frac{3}{z}\left(z w^{\prime} / 24\right) n^{\alpha / 4}=$ $w^{\prime} n^{\alpha / 4} / 8$ bids. For $n$ SL, this implies that the number of bids in $\left[J_{1}^{\lambda}-y / 2, J_{1}^{\lambda}\right]$ is at most $w^{\prime} y n / 4$ (by the same argument as in the proof of Lemma 3). Thus, given $\left\{\overline{c g} \in\left[J_{0}^{\lambda}, J_{4 / 5}^{\lambda}\right]\right\} \cap \mathcal{N}^{\prime}$, there are at least $w^{\prime} y n / 2-w^{\prime} y n / 4=w^{\prime} y n / 4$ players with value in $\left[J_{1}^{\lambda}-y / 2, J_{1}^{\lambda}\right]$ but bid below $\overline{c g}=J_{1}^{\lambda}-y$. But then,

$$
S L_{B}(y / 2) \geq \frac{w^{\prime} y n}{4} \frac{y}{2} \text {. }
$$

As $\overline{c g} \in\left[J_{0}^{\lambda}, J_{4 / 5}^{\lambda}\right], y \geq x / 5$. So, whenever $\left\{\overline{c g} \in\left[J_{0}^{\lambda}, J_{4 / 5}^{\kappa}\right]\right\} \cap \mathcal{N}^{\prime}$, 


$$
\begin{aligned}
S L_{B}(x / 10) & \geq S L_{B}(y / 2) \\
& \geq \frac{w^{\prime} n x^{2}}{200} .
\end{aligned}
$$

And, for $n \mathrm{SL}$,

$$
\begin{aligned}
\operatorname{Pr}\left(\left\{\overline{c g} \in\left[J_{0}^{\lambda}, J_{4 / 5}^{\kappa}\right]\right\} \cap \mathcal{N}^{\prime}\right) & \geq \operatorname{Pr}\left(\overline{c g} \in \cup_{\lambda}\left[J_{0}^{\lambda}, J_{4 / 5}^{\lambda}\right]\right)-\frac{1}{n^{4}} \\
& \geq \frac{1}{2} \operatorname{Pr}\left(\overline{c g} \in \cup_{\lambda}\left[J_{0}^{\lambda}, J_{4 / 5}^{\lambda}\right]\right) .
\end{aligned}
$$

Thus,

$$
E\left(S L_{B}\left(\frac{x}{10}\right)\right) \geq \frac{w^{\prime} n x^{2}}{400} \operatorname{Pr}\left(\overline{c g} \in \cup_{\lambda}\left[J_{0}^{\lambda}, J_{4 / 5}^{\lambda}\right]\right) .
$$

Now, for $n$ SL $\frac{x}{10}>\frac{1}{n}$, and hence $S L_{B}\left(\frac{x}{10}\right)<S L_{B}\left(\frac{1}{n}\right)$. However, $E\left(S L_{B}(1 / n)\right) \leq$ $\frac{1}{n^{1-\alpha_{3}}}$ by Lemma 8 . Thus,

$$
\frac{1}{n^{1-\alpha_{3}}} \geq \frac{w^{\prime} n x^{2}}{400} \operatorname{Pr}\left(\overline{c g} \in \cup_{\lambda}\left[J_{0}^{\lambda}, J_{4 / 5}^{\lambda}\right]\right) .
$$

Rearranging,

$$
\begin{aligned}
\operatorname{Pr}\left(\overline{c g} \in \cup_{\lambda}\left[J_{0}^{\lambda}, J_{4 / 5}^{\lambda}\right]\right) & \leq \frac{400}{n^{2-\alpha_{3}} x^{2}} \\
& \leq \frac{1}{12 n^{2-\alpha_{2}} x^{2}}
\end{aligned}
$$

for $n$ SL. Repeat for sellers in the lower fifth to get the second claim.

Step 4. Relative Probabilities of competitive and supporting gaps. Let $c g_{\lambda} \equiv$ $\left\{c g \supseteq\left[J_{1 / 5}^{\lambda}, J_{4 / 5}^{\lambda}\right]\right\}$, and let $c_{\lambda} \equiv \operatorname{Pr}\left(c g_{\lambda}\right)$. Similar, let $l g_{\lambda} \equiv\left\{l g \supseteq\left[J_{1 / 5}^{\lambda}, J_{2 / 5}^{\lambda}\right]\right\}$, and $l_{\lambda} \equiv \operatorname{Pr}\left(l g_{\lambda}\right)$. Finally, let $u g_{\lambda} \equiv\left\{u g \supseteq\left[J_{3 / 5}^{\lambda}, J_{4 / 5}^{\lambda}\right]\right\}$, and $u_{\lambda} \equiv \operatorname{Pr}\left(u g_{\lambda}\right)$. Our next lemma shows that for some $\lambda, c_{\lambda}$ is both non-trivial, and much larger than either $l_{\lambda}$ or $u_{\lambda}$.

Lemma 11 For $n S L$, there exists $\lambda$ such that

$$
c_{\lambda}>\frac{1}{n^{4}}
$$

and such that

$$
\frac{l_{\lambda}+u_{\lambda}}{c_{\lambda}} \leq \frac{4}{n^{\alpha_{2}-\alpha_{3}}}
$$


Proof As $\operatorname{Pr}\left(|c g|^{2} \geq x\right)>n^{\alpha_{2}-2} x^{-2}$ and $\operatorname{Pr}\left(\mathcal{N}^{\prime}\right) \geq 1-1 / n^{4}$, for $n$ SL $\operatorname{Pr}\left(\left\{|c g|^{2} \geq x\right\} \cap \mathcal{N}^{\prime}\right) \geq$ ${ }_{6}^{5} n^{\alpha_{2}-2} x^{-2}$. By Lemma 10, the probability of a competitive gap in $J^{\lambda}$ not including the middle $3 / 5$ is also less than $\frac{1}{6} n^{\alpha_{2}-2} x^{-2}$ for $n$ SL. Therefore, $\sum_{\lambda \in \Lambda} c_{\lambda} \geq \frac{4}{6} n_{t}^{\alpha_{2}-2} x_{t}^{-2}$. Let $\Lambda^{\prime}$ denote the subset of regions with $c_{\lambda}>1 / n^{4}$. There are at most $n$ regions. Thus,

$$
\sum_{\lambda \in \Lambda \backslash \Lambda^{\prime}} c_{\lambda} \leq \frac{n}{n^{4}} \leq \frac{1}{6} n^{\alpha_{2}-2} x^{-2}
$$

for $n$ SL. Thus

$$
\sum_{\lambda \in \Lambda^{\prime}} c_{\lambda} \geq \frac{1}{2} n^{\alpha_{2}-2} x^{-2}
$$

From Lemma 7, for $n$ SL $\sum_{\lambda \in \Lambda^{\prime}} l_{\lambda}+u_{\lambda} \leq 2 n^{\alpha_{3}-2} x^{-2}$. Thus

$$
\frac{\sum_{\lambda \in \Lambda^{\prime}} l_{\lambda}+u_{l}}{\sum_{\lambda \in \Lambda^{\prime}} c_{\lambda}} \leq \frac{2 n^{\alpha_{3}-2} x^{-2}}{\frac{1}{2} n^{\alpha_{2}-2} x^{-2}}=\frac{4}{n^{\alpha_{2}-\alpha_{3}}} .
$$

for $n \mathrm{SL}$. Since this is true on average, it must be true for at least one $\lambda \in \Lambda^{\prime}$

In what follows, we refer to a $\lambda$ for which Lemma 11 holds. Let $\tilde{c} \equiv \operatorname{Pr}(c g \supseteq$ $\left.\left[J_{2 / 5}^{\lambda}, J_{3 / 5}^{\lambda}\right]\right)$ be the probability of a competitive gap including the middle fifth of $J^{\lambda}$. We will show that $\tilde{c}$ is close to 1 . The idea is that the only way to have $c_{\lambda}$ be large relative to $u=u_{\lambda}$ and $l=l_{\lambda}$ will be for players to almost always get it almost right.

Let $U_{i} \equiv\left\{b_{i} \geq J_{4 / 5}^{\lambda}\right\}$ be the event that $i$ bids up and $\tilde{U}_{i} \equiv\left\{b_{i} \geq J_{2 / 5}^{\lambda}\right\}$ be the event that $i$ bids weakly up. Symmetrically, let $D_{i} \equiv\left\{b_{i} \leq J_{1 / 5}^{\lambda}\right\}$ and $\tilde{D}_{i} \equiv\left\{b_{i} \leq J_{3 / 5}^{\lambda}\right\}$ be the events that $i$ bids down and weakly down. Let $p_{i} \equiv \operatorname{Pr}\left(U_{i}\right), \tilde{p}_{i} \equiv \operatorname{Pr}\left(\tilde{U}_{i}\right), q_{i} \equiv \operatorname{Pr}\left(D_{i}\right)$ and $\tilde{q}_{i} \equiv \operatorname{Pr}\left(\tilde{D}_{i}\right)$. Order the players so that $\tilde{q}_{1} \leq \tilde{q}_{2} \leq \ldots \leq \tilde{q}_{2 n}$.

Step 5. A preliminary inequality. Define

$$
A_{-j}^{i} \equiv \cap_{j^{\prime}>i, j^{\prime} \neq j} D_{j}
$$

as the event that all players after $i$ not including $j$ bid down. Then, for any event $F$ involving $1,2, \ldots, i$,

$$
\begin{aligned}
\operatorname{Pr}\left(A_{-j}^{i} \mid F\right) & \leq \prod_{j^{\prime}>i, j^{\prime} \neq j} \operatorname{Pr}\left(D_{j^{\prime}} \mid D_{j^{\prime}-1}, \ldots, D_{i+1}, F\right) \\
& =\exp \left[\sum_{j^{\prime}>i, j^{\prime} \neq j} \log \operatorname{Pr}\left(D_{j^{\prime}} \mid D_{j^{\prime}-1}, \ldots, D_{i+1}, F\right)\right] \\
& \leq \exp \left[\sum_{j^{\prime}>i, j^{\prime} \neq j}\left(\operatorname{Pr}\left(D_{j^{\prime}} \mid D_{j^{\prime}-1}, \ldots, D_{i+1}, F\right)-1\right)\right] \quad(\text { since } \log x \leq x-1)
\end{aligned}
$$




$$
\begin{aligned}
& \leq \exp \left[-\sum_{j^{\prime}>i, j^{\prime} \neq j} \operatorname{Pr}\left(D_{j^{\prime}}^{c} \mid D_{j^{\prime}-1}, \ldots, D_{i+1}, F\right)\right] \\
& \leq e^{-z \sum_{j^{\prime}>i, j^{\prime} \neq j} 1-q_{j^{\prime}}}(\text { by } z \text {-independence }) \\
& \leq e^{-z \sum_{j^{\prime}>i, j^{\prime} \neq j} p_{j^{\prime}}}\left(\text { since } 1-q_{j^{\prime}} \geq p_{j^{\prime}}\right) \\
& \leq e^{-z\left(-1+\sum_{j^{\prime}>i} p_{j^{\prime}}\right)} .
\end{aligned}
$$

Step 6. Two Bounds. Recall that $c g_{\lambda} \equiv\left\{c g \supseteq\left[J_{1 / 5}^{\lambda}, J_{4 / 5}^{\lambda}\right]\right\}$. Let $c g_{\lambda}^{i j}, i<j$, be the event $c g_{\lambda}$ where $i$ and $j$ are the two highest indexed players for whom $U_{i}$ holds. Let $c_{\lambda}^{i j}=\operatorname{Pr}\left(c g_{\lambda}^{i j}\right)$. Let $F^{i}$ be the event that $U_{j^{\prime}}$ holds for $j^{\prime}=i$ and for $n-2$ other $j^{\prime} \in\{1, \ldots, i\}$, while $D_{j^{\prime}}$ holds for all other $j^{\prime} \in\{1, \ldots, i\}$. Then,

$$
\begin{aligned}
c_{\lambda}^{i j} & =\operatorname{Pr}\left(F^{i} \cap A_{-j}^{i} \cap U_{j}\right) \\
& =\operatorname{Pr}\left(U_{j} \mid F^{i} \cap A_{-j}^{i}\right) \operatorname{Pr}\left(F^{i} \cap A_{-j}^{i}\right) \\
& =\frac{\operatorname{Pr}\left(\tilde{D}_{j} \mid F^{i} \cap A_{-j}^{i}\right)}{\operatorname{Pr}\left(\tilde{D}_{j} \mid F^{i} \cap A_{-j}^{i}\right)} \operatorname{Pr}\left(U_{j} \mid F^{i} \cap A_{-j}^{i}\right) \operatorname{Pr}\left(F^{i} \cap A_{-j}^{i}\right) \\
& \leq \frac{p_{j}}{z^{2} \tilde{q}_{j}} \operatorname{Pr}\left(\tilde{D}_{j} \mid F^{i} \cap A_{-j}^{i}\right) \operatorname{Pr}\left(F^{i} \cap A_{-j}^{i}\right) \\
& =\frac{p_{j}}{z^{2} \tilde{q}_{j}} \operatorname{Pr}\left(\tilde{D}_{j} \cap F^{i} \cap A_{-j}^{i}\right) .
\end{aligned}
$$

Let $u g_{\lambda}^{i}$ be the event $u g_{\lambda}$, where $i$ is the last player to bid up, and let $u_{\lambda}^{i}=$ $\operatorname{Pr}\left(u g_{\lambda}^{i}\right)$. When $\tilde{D}_{j} \cap F^{i} \cap A_{-j}^{i}$ holds, $i$ is the last player to bid up and in total $n-1$ players bid up while the rest bid weakly down. Thus, $\left\{\tilde{D}_{j} \cap F^{i} \cap A_{-j}^{i}\right\} \subseteq u g_{\lambda}$, and so $\operatorname{Pr}\left(\tilde{D}_{j} \cap F^{i} \cap A_{-j}^{i}\right) \leq u_{\lambda}^{i}$. The previous equation thus implies

$$
c_{\lambda}^{i j} \leq \frac{p_{j}}{z^{2} \tilde{q}_{j}} u_{\lambda}^{i} .
$$

Another bound on $c_{\lambda}^{i j}$ comes from (30):

$$
c_{\lambda}^{i j} \leq \operatorname{Pr}\left(A_{-j}^{i}\right) \leq e^{-z\left(-1+\sum_{j^{\prime}>i} p_{j^{\prime}}\right)} .
$$

Step 7. Up and Down Players. We next show that for all $n \mathrm{SL}, \operatorname{Pr}\left(\tilde{D}_{i}\right) \leq \frac{1}{4}$ for $i \leq n$, and $\operatorname{Pr}\left(\tilde{U}_{i}\right) \geq \frac{1}{4}$ for $i>n$.

Consider the first claim. Suppose that $\tilde{q}_{n}>0$ (if $\tilde{q}_{n}=0$ the result is immediate). Let $c_{\lambda}^{i} \equiv \sum_{j>i} c_{\lambda}^{i j}$ be the probability that $c g_{\lambda}$ occurs, where $i$ is the second last up player. Let $i^{*} \leq n$ be the last index with the property that $\sum_{j^{\prime}>i} p_{j^{\prime}}>n^{\left(\alpha_{2}-\alpha_{3}\right) / 2}$. Then, 


$$
\begin{aligned}
\sum_{i \leq i^{*}} c_{\lambda}^{i} & =\sum_{i \leq i^{*} j>i} c_{\lambda}^{i j} \\
& \leq n^{2} e^{-z\left(-1+\sum_{j^{\prime}>i} p_{j^{\prime}}\right)}(\text { using }(32)) \\
& \leq n^{2} e^{-z\left(-1+n^{\left(\alpha_{2}-\alpha_{3}\right) / 2}\right)} \\
& \leq \frac{1}{2 n^{4}}(\text { for } n \mathrm{SL}) \\
& \leq \frac{1}{2} c_{\lambda}(\text { using }(28))
\end{aligned}
$$

So, as $c_{\lambda}=\sum_{i \geq n-1} c_{\lambda}^{i}$, for $n$ SL,

$$
\begin{aligned}
\frac{1}{2} c_{\lambda} & \leq \sum_{i>i^{*}, i \geq n-1} c_{\lambda}^{i} \\
& \leq \sum_{i>i^{*}, i \geq n-1} \sum_{j>i} \frac{p_{j}}{z^{2} \tilde{q}_{j}} u_{\lambda}^{i} \text { (using (31)) } \\
& \leq \frac{1}{z^{2} \tilde{q}_{n}} \sum_{i>i^{*}, i \geq n-1} \sum_{j>i} p_{j} u_{\lambda}^{i} \text { (since } \tilde{q}_{i} \text { is increasing) } \\
& \left.\leq \frac{n^{\left(\alpha_{2}-\alpha_{3}\right) / 2}}{z^{2} \tilde{q}_{n}} \sum_{i>i^{*}, i \geq n-1} u_{\lambda}^{i} \text { (by choice of } i^{*}\right) \\
& \leq \frac{n^{\left(\alpha_{2}-\alpha_{3}\right) / 2}}{z^{2} \tilde{q}_{n}} u_{\lambda} \\
& \left.\leq \frac{n^{\left(\alpha_{2}-\alpha_{3}\right) / 2}}{z^{2} \tilde{q}_{n}} \frac{4}{n^{\alpha_{2}-\alpha_{3}}} c_{\lambda} \text { (by }(29)\right) \\
& =\frac{4}{z^{2} \tilde{q}_{n}} \frac{1}{n^{\left(\alpha_{2}-\alpha_{3}\right) / 2}} c_{\lambda} .
\end{aligned}
$$

Comparing the first and last expressions, $\tilde{q}_{n} \rightarrow 0$, and so in particular, $q_{i} \leq 1 / 4$ all $i \leq n$ for all $n$ SL.

If the players are ordered so that $\tilde{p}_{i}$ increases, this argument can be repeated considering events in which $n-1$ of the first $i$ players bid down and the others bid up. Thus there are $n$ players for which $\tilde{p}_{i} \rightarrow 0$. As $\tilde{p}_{i}+\tilde{q}_{i} \geq 1$ these players are disjoint from players $1, \ldots, n$, and so must be the players $\{n+1, \ldots, 2 n\}$.

Let $R \equiv \cap_{i \leq n} U_{i} \cap_{i>n} D_{i}=c g_{\lambda}^{n-1, n}$ be the event that all the players bid according to their type (and a competitive gap occurs).

Step 8. A lower bound for $\operatorname{Pr}(R)$ : We already know that $\sum_{i \leq i^{*}, j>i} c_{\lambda}^{i j} \leq \frac{1}{2} c_{\lambda}$ for $n \mathrm{SL}$. We will show that for $n \mathrm{SL} \sum_{i>i^{*}, j>n+1} c_{\lambda}^{i j} \leq \frac{1}{4}$. Since $R$ is the only 
event left, it would then follow that $\operatorname{Pr}(R) \geq \frac{c^{4}}{4}$. So, as in Step 7 note that

$$
\begin{aligned}
\sum_{i>i^{*}, j \geq n+1} c_{\lambda}^{i j} & \leq \sum_{i>i^{*}, j \geq n+1} \frac{p_{j}}{z^{2} \tilde{q}_{j}} u_{\lambda}^{i}(\operatorname{using}(31)) \\
& \leq \frac{1}{z^{2} \tilde{q}_{n+1}} \sum_{i>i^{*}} u_{\lambda}^{i} \sum_{j>i} p_{j}(\text { note the } n+1) \\
& \leq \frac{n^{\left(\alpha_{2}-\alpha_{3}\right) / 2}}{2 z^{2}} \sum_{i>i^{*}} u_{\lambda}^{i}\left(\text { by choice of } i^{*}, \text { and since } \tilde{q}_{n+1} \cong 1\right) \\
& \leq \frac{n^{\left(\alpha_{2}-\alpha_{3}\right) / 2}}{2 z^{2}} u_{\lambda} \\
& \leq \frac{n^{\left(\alpha_{2}-\alpha_{3}\right) / 2}}{2 z^{2}} \frac{1}{n^{\alpha_{2}-\alpha_{3}}} c_{\lambda}(\text { by }(29)) \\
& =\frac{1}{2 z^{2}} \frac{1}{n^{\left(\alpha_{2}-\alpha_{3}\right) / 2}} c_{\lambda} \\
& \leq \frac{1}{4} c_{\lambda}(\text { for } n \mathrm{SL}) .
\end{aligned}
$$

Step 9. A Persistent Competitive Gap. Let $\tilde{R} \supset R$ be the event that all the players get it nearly right — the first $n$ players are not bidding below $J_{3 / 5}^{\lambda}$ and the others are not bidding above $J_{2 / 5}^{\lambda}$. For $i>n$, define $R_{-i}$ to be the event that all players except $i$ play according to type. If $R_{-i}$ occurs and player $i$ bids weakly up, then there will be a $l g \supset\left[J_{1 / 5}^{\lambda}, J_{2 / 5}^{\lambda}\right]$. Thus,

$$
\begin{aligned}
u_{\lambda} & \geq \sum_{i>n} \operatorname{Pr}\left(R_{-i}\right) \operatorname{Pr}\left(\tilde{U}_{i} \mid R_{-i}\right) \\
& \geq z \operatorname{Pr}(R) \sum_{i>n} \tilde{p}_{i}\left(\text { by z-independence and since } R \subseteq R_{-i}\right) \\
& \geq \frac{z c_{\lambda}}{4} \sum_{i>n} \tilde{p}_{i}
\end{aligned}
$$

Since $\frac{u_{\lambda}}{c_{\lambda}} \rightarrow 0$

$$
\sum_{i>n} \tilde{p}_{i} \rightarrow 0
$$

Arguing symmetrically,

$$
\sum_{i \leq n} \tilde{q}_{i} \rightarrow 0
$$

With the ordering described in Step 2, $\tilde{R}$ occurs when the first $n$ players do not bid weakly down and the last $n$ players do not bid weakly up, that is, $\tilde{R}=\left(\cap_{i \leq n} \tilde{D}_{i}^{c}\right) \cap\left(\cap_{i>n} \tilde{U}_{i}^{c}\right)$. Thus,

$$
\operatorname{Pr}(\tilde{R}) \geq 1-\sum_{i \leq n} \tilde{q}_{i}-\sum_{i>n} \tilde{p}_{i} \rightarrow 1 .
$$


Step 10. A Contradiction. When $\tilde{R} \cap \mathcal{N}^{\prime}$ occurs, $\left[J_{2 / 5}^{\lambda}, J_{3 / 5}^{\lambda}\right]$ is in the interior of $c g$ (players $1, \ldots, n$ bid strictly above $J_{3 / 5}$, and other players strictly below $J_{2 / 5}$. And since the probability of trade is bounded away from 0 , and since $\operatorname{Pr}(\tilde{R}) \rightarrow 1$, there is at least one buyer in $\{1, \ldots, n\}$ and at least one seller in $\{n+1, \ldots, 2 n\}$.

Let $p^{*}$ be the expected price conditional on $\tilde{R}$. Either $p^{*} \leq J_{1 / 2}^{\lambda}$, or $p^{*} \geq$ $J_{1 / 2}^{\lambda}$. Wlog, assume $p^{*} \geq J_{1 / 2}^{\lambda}$. Let $x^{\lambda}$ be the length of $J^{\lambda}$. By construction, $x^{\lambda} \geq \frac{1}{2} x \geq \frac{1}{2 n^{1-\alpha_{2} / 2}}$

Assume first that $J_{1}^{\lambda} \geq 1-3 x^{\lambda}$. Consider any buyer in $\{1, \ldots, n\}$. A bid of $J_{2 / 5}^{\lambda}$ wins whenever $\tilde{R}$ occurs, and forces the price to at most $J_{2 / 5}^{\lambda}$. So, conditional on $\tilde{R}$, the buyer's expected gain from lowering the price is at least $J_{1 / 2}^{\lambda}-J_{2 / 5}^{\lambda} \geq \frac{x^{\lambda}}{6}$. On the other hand, when $\tilde{R}$ does not occur, he may go from being a winner to a loser. But, for this to happen, it must be that $\underline{c g} \geq J_{2 / 5}^{\lambda}$. But then $i$ 's lost profit is at most $1-\underline{c g} \leq 4 x^{\lambda}$. Since $\operatorname{Pr}(\tilde{R}) \rightarrow 1$,

$$
\operatorname{Pr}(\tilde{R}) \frac{x^{\lambda}}{6}-(1-\operatorname{Pr}(\tilde{R})) 4 x^{\lambda}
$$

is eventually positive, and we have a contradiction.

Assume $J_{1}^{\lambda}<1-3 x^{\lambda}$. Given $\mathcal{N}^{\prime}$, the number of buyers with value in $\left(J_{1}^{\lambda}+2 x^{\lambda}, J_{1}^{\lambda}+3 x^{\lambda}\right)$ is at least $w^{\prime} n x^{\lambda}$. But, by Lemma 8 for $n$ SL

$$
E\left(\# U\left(x^{\lambda}\right)\right) \leq \frac{1}{n^{1-\alpha_{4} x^{\lambda}}} .
$$

It follows that for $n \mathrm{SL}$, at least half the buyers with value in $\left(J_{1}^{\lambda}+2 x^{\lambda}, J_{1}^{\lambda}+3 x^{\lambda}\right)$ trade conditional on $\tilde{R} \cap \mathcal{N}^{\prime}$ (and so bid above $J_{3 / 5}^{\lambda}$ ) Consider the deviation that any buyer with value in $\left(J_{1}^{\lambda}+2 x^{\lambda}, J_{1}^{\lambda}+3 x^{\lambda}\right)$ and bid above $J^{\lambda}$ bids $J_{2 / 5}^{\lambda}$ instead. Given $\tilde{R} \cap \mathcal{N}^{\prime}$, this gains the buyer at least $x^{\lambda} / 6$. Given $\mathcal{N}^{\prime}$, the number of players for in $\left(J_{1}^{\lambda}+2 x^{\lambda}, J_{1}^{\lambda}+3 x^{\lambda}\right)$ is at least $w^{\prime} n x^{\lambda}$ and at most $W^{\prime} n x^{\lambda}$. So, given $R \cap \mathcal{N}^{\prime}$, the expected sum of gains is at least $\frac{w^{\prime} n x^{\lambda}}{2} \frac{x^{\lambda}}{6}$. The loss from such a buyer going from being a winner to a loser is again at most $4 x^{\lambda}$. Given $\mathcal{N}^{\prime} \backslash \tilde{R}$, there are at most $W^{\prime} n x^{\lambda}$ such buyers. In $\mathcal{N}^{\prime c}$, the worst case is that all $n$ buyers are in $\left(J_{1}^{\lambda}+2 x^{\lambda}, J_{1}^{\lambda}+3 x^{\lambda}\right)$. So, the expected sum of losses is at most

$$
\begin{aligned}
& \operatorname{Pr}\left(\mathcal{N}^{\prime} \backslash \tilde{R}\right) W^{\prime} n x^{\lambda} 4 x^{\lambda}+\operatorname{Pr}\left(\mathcal{N}^{\prime c}\right) n 4 x^{\lambda} \\
\leq & (1-\operatorname{Pr}(\tilde{R})) W^{\prime} n x^{\lambda} 4 x^{\lambda}+\frac{1}{n^{4}} n 4 x^{\lambda}
\end{aligned}
$$

and thus, since the deviation cannot be profitable,

$$
\operatorname{Pr}(\tilde{R}) \frac{w^{\prime} n x^{\lambda}}{2} \frac{x^{\lambda}}{6} \leq(1-\operatorname{Pr}(\tilde{R})) W^{\prime} n x^{\lambda} 4 x^{\lambda}+\frac{1}{n^{4}} n 4 x^{\lambda} .
$$


Dividing both sides by $n\left(x^{\lambda}\right)^{2}$,

$$
\begin{aligned}
\operatorname{Pr}(\tilde{R}) \frac{w^{\prime}}{12} & \leq 4 W^{\prime}(1-\operatorname{Pr}(\tilde{R}))+\frac{4}{n^{2} x^{\lambda}} \\
& \leq 4 W^{\prime}(1-\operatorname{Pr}(\tilde{R}))+\frac{8}{n^{2} x}\left(\text { since } x^{\lambda} \geq \frac{1}{2} x\right) \\
& \leq 4 W^{\prime}(1-\operatorname{Pr}(\tilde{R}))+\frac{8}{n^{2} \frac{1}{n^{1-\alpha_{2} / 2}}} \\
& =4 W^{\prime}(1-\operatorname{Pr}(\tilde{R}))+\frac{8}{n^{1+\alpha_{2} / 2}} .
\end{aligned}
$$

The LHS goes to $w^{\prime} / 12$, while the RHS goes to 0 , a contradiction.

\subsection{Proofs for Section 5}

Let $x$ be the random variable $\overline{s g}_{m}-\underline{c g}$. In an $m$ unit demand/supply setting, this is the maximum impact of raising a buyer's bid vector on price. Let $p$ be the price. We will show that in expectation buyers achieve within $\frac{1}{2 n^{1-\alpha}}$ of the consumer surplus if they can price take at $p$. A symmetric argument applies to sellers. But, the sum of consumer and producer surplus at an arbitrary $p$ is at least as large as the surplus at the Walrasian price. So, this both establishes that the market achieves within $1 / n^{1-\alpha}$ of the efficient surplus and that price must be asymptotically Walrasian (else the market achieves more than the feasible surplus, a contradiction). Finally, from NAG and NAA, expected feasible surplus grows like $n$, and the result follows.

Consider the truth-telling deviation $d_{i}\left(b_{i}, v_{i}\right)=v_{i}$, remembering that $v_{i}$ and $b_{i}$ are now vectors in $[0,1]^{m}$. Let $W$ be the set of $i h, i \in N_{B}$ that are allocated an object. Let $S L_{i h}=0$ if $i$ wins an object $h$, and let $S L_{i h}=\max \left[v_{i h}-p, 0\right]$ other wise. So, $S L_{i h}$ gives the loss in consumer surplus compared to taking at $p$ from $i$ not winning object $h$.

In any given realization, think about moving from $b_{i}$ to $v_{i}$ one bid at a time, starting from $b_{i 1}$. Let $\hat{C}_{i h}$ be the cost to $i$ from raising bid $h$ in terms of raising the price paid on units already won, and $\hat{B}_{i h}$ the profit to $i$ of winning an extra unit.

If $v_{i h}<\underline{c g}$, then raising $b_{i h}$ to $v_{i h}$ is irrelevant to both $p$ and the allocation. Hence, $\hat{B}_{i h}-\hat{C}_{i h}=0$. And, since $v_{i h}<p, S L_{i h}=0$.

If $v_{i h} \in[c g, \overline{s g}+2 m x]$, then raising $b_{i h}$ to $v_{i h}$ may raise the price on units already won by as much as $x$. So, $\hat{B}_{i h}-\hat{C}_{i h} \geq-(m-1) x$. And, since $v_{i h} \in$ $[\underline{c g}, \overline{s g}+2 m x]$ and $p \geq \underline{c g}, S L_{i h} \leq(2 m+1) x$. In any normal realization, the number of such ih is at most $K n x$ for some $K<\infty$. In a non-normal realization, there are at most $n m$ values in this range. Hence, the expected number of such values is at most

$$
\begin{aligned}
& \left(1-\frac{1}{n^{4}}\right) K n x+\frac{1}{n^{4}} n m \\
\leq & K n x+\frac{m}{n^{3}}
\end{aligned}
$$


Consider $i h \in W$ such that $v_{i h}>\overline{s g}+2 m x$. In any realization, at most 1 player who is winning an object is also in a position to affect the price by changing the associated bid. And, the impact of that bid on price is at most $x$. Hence,

$$
\sum_{\left\{i h \in W \mid v_{i h}>\overline{s g}+2 m x\right\}} \hat{C}_{i h} \leq x
$$

and

$$
\sum_{\left\{i h \in W \mid v_{i h}>\overline{s g}+2 m x\right\}} S L_{i h}=0 .
$$

Finally, consider $i h \notin W$ such that $v_{i h}>\overline{s g}+2 m x$. Then, by deviating to $b_{i h}=v_{i h}, i$ raises the raise the price on at most $m-1$ previous units by at most $x$. But, $i$ also wins an extra object at price at most $\overline{s g}$. So,

$$
\hat{B}_{i h}-\hat{C}_{i h} \geq v_{i h}-\overline{s g}-(m-1) x \geq \frac{v_{i h}-p}{2}=\frac{S L_{i h}}{2} .
$$

Since we are in equilibrium

$$
\begin{aligned}
0 \geq & E\left(\sum_{i h} \hat{B}_{i h}-\hat{C}_{i h}\right) \\
= & E\left(E\left(\sum_{\left\{i h \notin W \mid v_{i h}>\overline{s g}+2 m x\right\}} \hat{B}_{i h}-\hat{C}_{i h} \mid x\right)\right)+E\left(E\left(\sum_{\left\{i h \in W \mid v_{i h}>\overline{s g}+2 m x\right\}} \hat{B}_{i h}-\hat{C}_{i h} \mid x\right)\right) \\
& +E\left(E\left(\sum_{\left\{i h \mid v_{i h} \in[\underline{c g}, \overline{s g}+2 m x]\right\}} \hat{B}_{i h}-\hat{C}_{i h} \mid x\right)\right) \\
\geq & E\left(E\left(\sum_{\left\{i h \mid v_{i h}>\overline{s g}+2 m x\right\}} \frac{S L_{i h} \mid}{2} \mid x\right)\right)-E(x)-E\left(E\left(\sum_{\left.\left\{i h \mid v_{i h} \in \underline{[c g}, \overline{s g}+2 m x\right]\right\}}(m-1) x \mid x\right)\right) \\
\geq & E\left(E\left(\sum_{\left\{i h \mid v_{i h}>\overline{s g}+2 m x\right\}} \frac{S L_{i h} \mid}{2} \mid x\right)\right)-E(x)-E\left(\left(K n x+\frac{m}{n^{3}}\right)(m-1) x\right) \\
\geq & E\left(E\left(\sum_{\left\{i h \mid v_{i h}>\overline{s g}+2 m x\right\}} \frac{S L_{i h} \mid}{2} \mid x\right)\right)-2 E(x)-K^{\prime \prime} n E\left(x^{2}\right)(\text { for } n \text { SL })
\end{aligned}
$$

Let $H$ be the cumulative for $x$. By Lemmas 7 and $9, H(x) \leq \frac{1}{n^{2-\alpha_{2} x^{2}}}$ for all 
$x>\frac{1}{n}$. Hence,

$$
\begin{aligned}
n E\left(x^{2}\right) & =\int_{0}^{1} n x^{2} d H(x) \\
& =\int_{0}^{1} n 2 x[1-H(x)] d x \\
& \leq 0+\int_{0}^{1 / n} 2 n x d x+\int_{1 / n}^{1} 2 n x \frac{1}{n^{2-\alpha_{2}} x^{2}} d x \\
& =\left.n x^{2}\right|_{0} ^{1 / n}+\frac{2}{n^{1-\alpha_{2}}} \int_{1 / n}^{1} \frac{1}{x} d x \\
& \leq \frac{1+2 \log n}{n^{1-\alpha_{2}}} \\
& \leq \frac{1}{n^{1-\alpha_{1}}}(\text { for } n \mathrm{SL}) .
\end{aligned}
$$

And, $E(x) \leq \frac{1}{n^{1-\alpha_{1}}}$ as well (a simple integration by parts). So, (33) yields

$$
\begin{aligned}
E\left(E\left(\sum_{\left\{i h \mid v_{i h}>\overline{s g}+2 m x\right\}} S L_{i h} \mid x\right)\right) & \leq 2\left(\frac{1}{n^{1-\alpha}}+K^{\prime \prime} \frac{1}{n^{1-\alpha}}\right) \\
& =K^{\prime \prime \prime} \frac{1}{n^{1-\alpha}}
\end{aligned}
$$

But then,

$$
\begin{aligned}
E\left(\sum_{i h} S L_{i h}\right) & =E\left(E\left(\sum_{\left\{i h \mid v_{i h}>\overline{s g}+2 m x\right\}} S L_{i h} \mid x\right)\right)+E\left(E\left(\sum_{v_{i h} \in[\underline{c}, \overline{s g}+2 m x]} S L_{i h} \mid x\right)\right) \\
& \leq K^{\prime \prime \prime} \frac{1}{n^{1-\alpha_{1}}}+E\left(K n x+\frac{m}{n^{3}}\right)(2 m+1) x \\
& \leq K^{\prime \prime \prime} \frac{1}{n^{1-\alpha_{1}}}+\frac{2 m+1}{n^{3}} x m E(x)+K^{\prime \prime \prime \prime} E\left(n x^{2}\right) \\
& \leq \frac{1}{2 n^{1-\alpha}} \text { for } n S L
\end{aligned}
$$

Arguing analogously for sellers, $E\left(\sum_{i h} S L_{i h}\right) \leq \frac{1}{2 n^{1-\alpha}}$. Hence, the expected sum of consumer and producer surplus is within $1 / n^{1-\alpha}$ of that achieved by the Walrasian outcome, and we are done. 